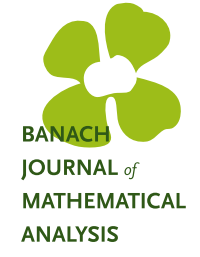

Banach J. Math. Anal. 10 (2016), no. 3, 638-670

http://dx.doi.org/10.1215/17358787-3639712

ISSN: $1735-8787$ (electronic)

http://projecteuclid.org/bjma

\title{
EQUATIONS FOR FRAME WAVELETS IN $L^{2}\left(\mathbb{R}^{2}\right)$
}

\author{
XINGDE DAI \\ To Professor Zurui Guo \\ Communicated by D. Han
}

\begin{abstract}
A finite solution to a system of equations will generate a single function normalized tight frame wavelet (Parseval's frame wavelet) with compact support associated with a $2 \times 2$ expansive integral matrix whose determinant is either 2 or -2 in $L^{2}\left(\mathbb{R}^{2}\right)$.
\end{abstract}

\section{IntRoduction}

In this article, $\mathbb{R}^{2}$ will be the 2-dimensional Euclidean space, and $\mathbb{C}^{2}$ will be the 2-dimensional complex Euclidean space. We will use notation $\vec{t}, \vec{s}, \vec{r}, \vec{\xi}, \vec{\eta}$ for vectors in $\mathbb{R}^{2}$ or $\mathbb{C}^{2}$. We will use the notation $\vec{t}_{1} \circ \vec{t}_{2}$ for the standard inner product of two vectors $\vec{t}_{1}$ and $\vec{t}_{2}$. For a vector $\vec{\xi}=\left(\begin{array}{c}\xi_{1} \\ \xi_{2}\end{array}\right)$ in $\mathbb{C}^{2}$, its real part is $\mathfrak{R e}(\vec{\xi}) \equiv\left(\begin{array}{c}\mathfrak{R e}\left(\xi_{1}\right) \\ \mathfrak{R}\left(\xi_{2}\right)\end{array}\right)$, and its imaginary part is $\mathfrak{I m}(\vec{\xi}) \equiv\left(\begin{array}{c}\mathfrak{I m}\left(\xi_{1}\right) \\ \mathfrak{I m}\left(\xi_{2}\right)\end{array}\right)$. The measure $\mu$ will be the Lebesgue measure on $\mathbb{R}^{2}$, and $L^{2}\left(\mathbb{R}^{2}\right)$ will be the Hilbert space of all square integrable functions on $\mathbb{R}^{2}$. A (countable) set of elements $\left\{\psi_{i}: i \in \Lambda\right\}$ in $L^{2}\left(\mathbb{R}^{2}\right)$ is called a normalized tight frame of $L^{2}\left(\mathbb{R}^{2}\right)$ if

$$
\sum_{i \in \Lambda}\left|\left\langle f, \psi_{i}\right\rangle\right|^{2}=\|f\|^{2}, \quad \forall f \in L^{2}\left(\mathbb{R}^{2}\right) .
$$

It is well known in the literature (see [4]) that the equation (1.1) is equivalent to

$$
f=\sum_{i \in \Lambda}\left\langle f, \psi_{i}\right\rangle \psi_{i}, \quad \forall f \in L^{2}\left(\mathbb{R}^{2}\right) .
$$

Copyright 2016 by the Tusi Mathematical Research Group.

Received Sep. 21, 2015; Accepted Dec. 15, 2015.

2010 Mathematics Subject Classification. Primary 46N99; Secondary 47N99, 46E99, 42C40.

Keywords. Fourier transform, Hilbert space, expansive integral matrix, normalized tight frame wavelet. 


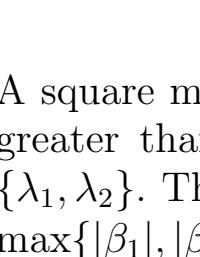

Banach J. Math. Anal. 10 (2016), no. 3, 638-670

http://dx.doi.org/10.1215/17358787-3639712

ISSN: $1735-8787$ (electronic)

http://projecteuclid.org/bjma

\title{
EQUATIONS FOR FRAME WAVELETS IN $L^{2}\left(\mathbb{R}^{2}\right)$
}

\author{
XINGDE DAI \\ To Professor Zurui Guo \\ Communicated by D. Han
}

\begin{abstract}
A finite solution to a system of equations will generate a single function normalized tight frame wavelet (Parseval's frame wavelet) with compact support associated with a $2 \times 2$ expansive integral matrix whose determinant is either 2 or -2 in $L^{2}\left(\mathbb{R}^{2}\right)$.
\end{abstract}

\section{IntRoduction}

In this article, $\mathbb{R}^{2}$ will be the 2-dimensional Euclidean space, and $\mathbb{C}^{2}$ will be the 2-dimensional complex Euclidean space. We will use notation $\vec{t}, \vec{s}, \vec{r}, \vec{\xi}, \vec{\eta}$ for vectors in $\mathbb{R}^{2}$ or $\mathbb{C}^{2}$. We will use the notation $\vec{t}_{1} \circ \vec{t}_{2}$ for the standard inner product of two vectors $\vec{t}_{1}$ and $\vec{t}_{2}$. For a vector $\vec{\xi}=\left(\begin{array}{c}\xi_{1} \\ \xi_{2}\end{array}\right)$ in $\mathbb{C}^{2}$, its real part is $\mathfrak{R e}(\vec{\xi}) \equiv\left(\begin{array}{c}\mathfrak{R e}\left(\xi_{1}\right) \\ \mathfrak{R}\left(\xi_{2}\right)\end{array}\right)$, and its imaginary part is $\mathfrak{I m}(\vec{\xi}) \equiv\left(\begin{array}{c}\mathfrak{I m}\left(\xi_{1}\right) \\ \mathfrak{I m}\left(\xi_{2}\right)\end{array}\right)$. The measure $\mu$ will be the Lebesgue measure on $\mathbb{R}^{2}$, and $L^{2}\left(\mathbb{R}^{2}\right)$ will be the Hilbert space of all square integrable functions on $\mathbb{R}^{2}$. A (countable) set of elements $\left\{\psi_{i}: i \in \Lambda\right\}$ in $L^{2}\left(\mathbb{R}^{2}\right)$ is called a normalized tight frame of $L^{2}\left(\mathbb{R}^{2}\right)$ if

$$
\sum_{i \in \Lambda}\left|\left\langle f, \psi_{i}\right\rangle\right|^{2}=\|f\|^{2}, \quad \forall f \in L^{2}\left(\mathbb{R}^{2}\right) .
$$

It is well known in the literature (see [4]) that the equation (1.1) is equivalent to

$$
f=\sum_{i \in \Lambda}\left\langle f, \psi_{i}\right\rangle \psi_{i}, \quad \forall f \in L^{2}\left(\mathbb{R}^{2}\right) .
$$

Copyright 2016 by the Tusi Mathematical Research Group.

Received Sep. 21, 2015; Accepted Dec. 15, 2015.

2010 Mathematics Subject Classification. Primary 46N99; Secondary 47N99, 46E99, 42C40.

Keywords. Fourier transform, Hilbert space, expansive integral matrix, normalized tight frame wavelet. 


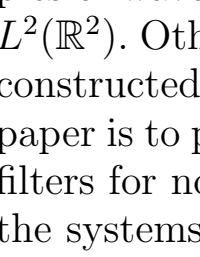

Banach J. Math. Anal. 10 (2016), no. 3, 638-670

http://dx.doi.org/10.1215/17358787-3639712

ISSN: $1735-8787$ (electronic)

http://projecteuclid.org/bjma

\title{
EQUATIONS FOR FRAME WAVELETS IN $L^{2}\left(\mathbb{R}^{2}\right)$
}

\author{
XINGDE DAI \\ To Professor Zurui Guo \\ Communicated by D. Han
}

\begin{abstract}
A finite solution to a system of equations will generate a single function normalized tight frame wavelet (Parseval's frame wavelet) with compact support associated with a $2 \times 2$ expansive integral matrix whose determinant is either 2 or -2 in $L^{2}\left(\mathbb{R}^{2}\right)$.
\end{abstract}

\section{IntRoduction}

In this article, $\mathbb{R}^{2}$ will be the 2-dimensional Euclidean space, and $\mathbb{C}^{2}$ will be the 2-dimensional complex Euclidean space. We will use notation $\vec{t}, \vec{s}, \vec{r}, \vec{\xi}, \vec{\eta}$ for vectors in $\mathbb{R}^{2}$ or $\mathbb{C}^{2}$. We will use the notation $\vec{t}_{1} \circ \vec{t}_{2}$ for the standard inner product of two vectors $\vec{t}_{1}$ and $\vec{t}_{2}$. For a vector $\vec{\xi}=\left(\begin{array}{c}\xi_{1} \\ \xi_{2}\end{array}\right)$ in $\mathbb{C}^{2}$, its real part is $\mathfrak{R e}(\vec{\xi}) \equiv\left(\begin{array}{c}\mathfrak{R e}\left(\xi_{1}\right) \\ \mathfrak{R}\left(\xi_{2}\right)\end{array}\right)$, and its imaginary part is $\mathfrak{I m}(\vec{\xi}) \equiv\left(\begin{array}{c}\mathfrak{I m}\left(\xi_{1}\right) \\ \mathfrak{I m}\left(\xi_{2}\right)\end{array}\right)$. The measure $\mu$ will be the Lebesgue measure on $\mathbb{R}^{2}$, and $L^{2}\left(\mathbb{R}^{2}\right)$ will be the Hilbert space of all square integrable functions on $\mathbb{R}^{2}$. A (countable) set of elements $\left\{\psi_{i}: i \in \Lambda\right\}$ in $L^{2}\left(\mathbb{R}^{2}\right)$ is called a normalized tight frame of $L^{2}\left(\mathbb{R}^{2}\right)$ if

$$
\sum_{i \in \Lambda}\left|\left\langle f, \psi_{i}\right\rangle\right|^{2}=\|f\|^{2}, \quad \forall f \in L^{2}\left(\mathbb{R}^{2}\right) .
$$

It is well known in the literature (see [4]) that the equation (1.1) is equivalent to

$$
f=\sum_{i \in \Lambda}\left\langle f, \psi_{i}\right\rangle \psi_{i}, \quad \forall f \in L^{2}\left(\mathbb{R}^{2}\right) .
$$

Copyright 2016 by the Tusi Mathematical Research Group.

Received Sep. 21, 2015; Accepted Dec. 15, 2015.

2010 Mathematics Subject Classification. Primary 46N99; Secondary 47N99, 46E99, 42C40.

Keywords. Fourier transform, Hilbert space, expansive integral matrix, normalized tight frame wavelet. 


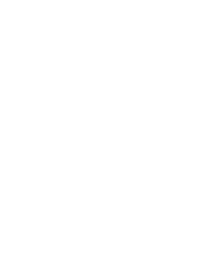

Banach J. Math. Anal. 10 (2016), no. 3, 638-670

http://dx.doi.org/10.1215/17358787-3639712

ISSN: $1735-8787$ (electronic)

http://projecteuclid.org/bjma

\title{
EQUATIONS FOR FRAME WAVELETS IN $L^{2}\left(\mathbb{R}^{2}\right)$
}

\author{
XINGDE DAI \\ To Professor Zurui Guo \\ Communicated by D. Han
}

\begin{abstract}
A finite solution to a system of equations will generate a single function normalized tight frame wavelet (Parseval's frame wavelet) with compact support associated with a $2 \times 2$ expansive integral matrix whose determinant is either 2 or -2 in $L^{2}\left(\mathbb{R}^{2}\right)$.
\end{abstract}

\section{IntRoduction}

In this article, $\mathbb{R}^{2}$ will be the 2-dimensional Euclidean space, and $\mathbb{C}^{2}$ will be the 2-dimensional complex Euclidean space. We will use notation $\vec{t}, \vec{s}, \vec{r}, \vec{\xi}, \vec{\eta}$ for vectors in $\mathbb{R}^{2}$ or $\mathbb{C}^{2}$. We will use the notation $\vec{t}_{1} \circ \vec{t}_{2}$ for the standard inner product of two vectors $\vec{t}_{1}$ and $\vec{t}_{2}$. For a vector $\vec{\xi}=\left(\begin{array}{c}\xi_{1} \\ \xi_{2}\end{array}\right)$ in $\mathbb{C}^{2}$, its real part is $\mathfrak{R e}(\vec{\xi}) \equiv\left(\begin{array}{c}\mathfrak{R e}\left(\xi_{1}\right) \\ \mathfrak{R}\left(\xi_{2}\right)\end{array}\right)$, and its imaginary part is $\mathfrak{I m}(\vec{\xi}) \equiv\left(\begin{array}{c}\mathfrak{I m}\left(\xi_{1}\right) \\ \mathfrak{I m}\left(\xi_{2}\right)\end{array}\right)$. The measure $\mu$ will be the Lebesgue measure on $\mathbb{R}^{2}$, and $L^{2}\left(\mathbb{R}^{2}\right)$ will be the Hilbert space of all square integrable functions on $\mathbb{R}^{2}$. A (countable) set of elements $\left\{\psi_{i}: i \in \Lambda\right\}$ in $L^{2}\left(\mathbb{R}^{2}\right)$ is called a normalized tight frame of $L^{2}\left(\mathbb{R}^{2}\right)$ if

$$
\sum_{i \in \Lambda}\left|\left\langle f, \psi_{i}\right\rangle\right|^{2}=\|f\|^{2}, \quad \forall f \in L^{2}\left(\mathbb{R}^{2}\right) .
$$

It is well known in the literature (see [4]) that the equation (1.1) is equivalent to

$$
f=\sum_{i \in \Lambda}\left\langle f, \psi_{i}\right\rangle \psi_{i}, \quad \forall f \in L^{2}\left(\mathbb{R}^{2}\right) .
$$

Copyright 2016 by the Tusi Mathematical Research Group.

Received Sep. 21, 2015; Accepted Dec. 15, 2015.

2010 Mathematics Subject Classification. Primary 46N99; Secondary 47N99, 46E99, 42C40.

Keywords. Fourier transform, Hilbert space, expansive integral matrix, normalized tight frame wavelet. 


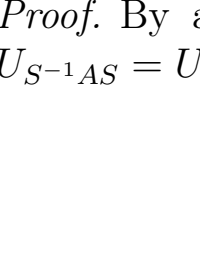

Banach J. Math. Anal. 10 (2016), no. 3, 638-670

http://dx.doi.org/10.1215/17358787-3639712

ISSN: $1735-8787$ (electronic)

http://projecteuclid.org/bjma

\title{
EQUATIONS FOR FRAME WAVELETS IN $L^{2}\left(\mathbb{R}^{2}\right)$
}

\author{
XINGDE DAI \\ To Professor Zurui Guo \\ Communicated by D. Han
}

\begin{abstract}
A finite solution to a system of equations will generate a single function normalized tight frame wavelet (Parseval's frame wavelet) with compact support associated with a $2 \times 2$ expansive integral matrix whose determinant is either 2 or -2 in $L^{2}\left(\mathbb{R}^{2}\right)$.
\end{abstract}

\section{IntRoduction}

In this article, $\mathbb{R}^{2}$ will be the 2-dimensional Euclidean space, and $\mathbb{C}^{2}$ will be the 2-dimensional complex Euclidean space. We will use notation $\vec{t}, \vec{s}, \vec{r}, \vec{\xi}, \vec{\eta}$ for vectors in $\mathbb{R}^{2}$ or $\mathbb{C}^{2}$. We will use the notation $\vec{t}_{1} \circ \vec{t}_{2}$ for the standard inner product of two vectors $\vec{t}_{1}$ and $\vec{t}_{2}$. For a vector $\vec{\xi}=\left(\begin{array}{c}\xi_{1} \\ \xi_{2}\end{array}\right)$ in $\mathbb{C}^{2}$, its real part is $\mathfrak{R e}(\vec{\xi}) \equiv\left(\begin{array}{c}\mathfrak{R e}\left(\xi_{1}\right) \\ \mathfrak{R}\left(\xi_{2}\right)\end{array}\right)$, and its imaginary part is $\mathfrak{I m}(\vec{\xi}) \equiv\left(\begin{array}{c}\mathfrak{I m}\left(\xi_{1}\right) \\ \mathfrak{I m}\left(\xi_{2}\right)\end{array}\right)$. The measure $\mu$ will be the Lebesgue measure on $\mathbb{R}^{2}$, and $L^{2}\left(\mathbb{R}^{2}\right)$ will be the Hilbert space of all square integrable functions on $\mathbb{R}^{2}$. A (countable) set of elements $\left\{\psi_{i}: i \in \Lambda\right\}$ in $L^{2}\left(\mathbb{R}^{2}\right)$ is called a normalized tight frame of $L^{2}\left(\mathbb{R}^{2}\right)$ if

$$
\sum_{i \in \Lambda}\left|\left\langle f, \psi_{i}\right\rangle\right|^{2}=\|f\|^{2}, \quad \forall f \in L^{2}\left(\mathbb{R}^{2}\right) .
$$

It is well known in the literature (see [4]) that the equation (1.1) is equivalent to

$$
f=\sum_{i \in \Lambda}\left\langle f, \psi_{i}\right\rangle \psi_{i}, \quad \forall f \in L^{2}\left(\mathbb{R}^{2}\right) .
$$

Copyright 2016 by the Tusi Mathematical Research Group.

Received Sep. 21, 2015; Accepted Dec. 15, 2015.

2010 Mathematics Subject Classification. Primary 46N99; Secondary 47N99, 46E99, 42C40.

Keywords. Fourier transform, Hilbert space, expansive integral matrix, normalized tight frame wavelet. 


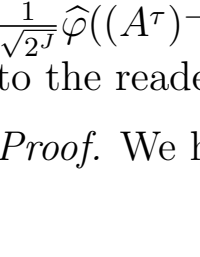

Banach J. Math. Anal. 10 (2016), no. 3, 638-670

http://dx.doi.org/10.1215/17358787-3639712

ISSN: $1735-8787$ (electronic)

http://projecteuclid.org/bjma

\title{
EQUATIONS FOR FRAME WAVELETS IN $L^{2}\left(\mathbb{R}^{2}\right)$
}

\author{
XINGDE DAI \\ To Professor Zurui Guo \\ Communicated by D. Han
}

\begin{abstract}
A finite solution to a system of equations will generate a single function normalized tight frame wavelet (Parseval's frame wavelet) with compact support associated with a $2 \times 2$ expansive integral matrix whose determinant is either 2 or -2 in $L^{2}\left(\mathbb{R}^{2}\right)$.
\end{abstract}

\section{IntRoduction}

In this article, $\mathbb{R}^{2}$ will be the 2-dimensional Euclidean space, and $\mathbb{C}^{2}$ will be the 2-dimensional complex Euclidean space. We will use notation $\vec{t}, \vec{s}, \vec{r}, \vec{\xi}, \vec{\eta}$ for vectors in $\mathbb{R}^{2}$ or $\mathbb{C}^{2}$. We will use the notation $\vec{t}_{1} \circ \vec{t}_{2}$ for the standard inner product of two vectors $\vec{t}_{1}$ and $\vec{t}_{2}$. For a vector $\vec{\xi}=\left(\begin{array}{c}\xi_{1} \\ \xi_{2}\end{array}\right)$ in $\mathbb{C}^{2}$, its real part is $\mathfrak{R e}(\vec{\xi}) \equiv\left(\begin{array}{c}\mathfrak{R e}\left(\xi_{1}\right) \\ \mathfrak{R}\left(\xi_{2}\right)\end{array}\right)$, and its imaginary part is $\mathfrak{I m}(\vec{\xi}) \equiv\left(\begin{array}{c}\mathfrak{I m}\left(\xi_{1}\right) \\ \mathfrak{I m}\left(\xi_{2}\right)\end{array}\right)$. The measure $\mu$ will be the Lebesgue measure on $\mathbb{R}^{2}$, and $L^{2}\left(\mathbb{R}^{2}\right)$ will be the Hilbert space of all square integrable functions on $\mathbb{R}^{2}$. A (countable) set of elements $\left\{\psi_{i}: i \in \Lambda\right\}$ in $L^{2}\left(\mathbb{R}^{2}\right)$ is called a normalized tight frame of $L^{2}\left(\mathbb{R}^{2}\right)$ if

$$
\sum_{i \in \Lambda}\left|\left\langle f, \psi_{i}\right\rangle\right|^{2}=\|f\|^{2}, \quad \forall f \in L^{2}\left(\mathbb{R}^{2}\right) .
$$

It is well known in the literature (see [4]) that the equation (1.1) is equivalent to

$$
f=\sum_{i \in \Lambda}\left\langle f, \psi_{i}\right\rangle \psi_{i}, \quad \forall f \in L^{2}\left(\mathbb{R}^{2}\right) .
$$

Copyright 2016 by the Tusi Mathematical Research Group.

Received Sep. 21, 2015; Accepted Dec. 15, 2015.

2010 Mathematics Subject Classification. Primary 46N99; Secondary 47N99, 46E99, 42C40.

Keywords. Fourier transform, Hilbert space, expansive integral matrix, normalized tight frame wavelet. 


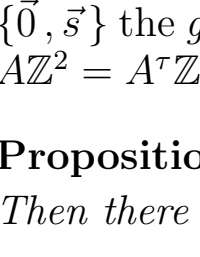

Banach J. Math. Anal. 10 (2016), no. 3, 638-670

http://dx.doi.org/10.1215/17358787-3639712

ISSN: $1735-8787$ (electronic)

http://projecteuclid.org/bjma

\title{
EQUATIONS FOR FRAME WAVELETS IN $L^{2}\left(\mathbb{R}^{2}\right)$
}

\author{
XINGDE DAI \\ To Professor Zurui Guo \\ Communicated by D. Han
}

\begin{abstract}
A finite solution to a system of equations will generate a single function normalized tight frame wavelet (Parseval's frame wavelet) with compact support associated with a $2 \times 2$ expansive integral matrix whose determinant is either 2 or -2 in $L^{2}\left(\mathbb{R}^{2}\right)$.
\end{abstract}

\section{IntRoduction}

In this article, $\mathbb{R}^{2}$ will be the 2-dimensional Euclidean space, and $\mathbb{C}^{2}$ will be the 2-dimensional complex Euclidean space. We will use notation $\vec{t}, \vec{s}, \vec{r}, \vec{\xi}, \vec{\eta}$ for vectors in $\mathbb{R}^{2}$ or $\mathbb{C}^{2}$. We will use the notation $\vec{t}_{1} \circ \vec{t}_{2}$ for the standard inner product of two vectors $\vec{t}_{1}$ and $\vec{t}_{2}$. For a vector $\vec{\xi}=\left(\begin{array}{c}\xi_{1} \\ \xi_{2}\end{array}\right)$ in $\mathbb{C}^{2}$, its real part is $\mathfrak{R e}(\vec{\xi}) \equiv\left(\begin{array}{c}\mathfrak{R e}\left(\xi_{1}\right) \\ \mathfrak{R}\left(\xi_{2}\right)\end{array}\right)$, and its imaginary part is $\mathfrak{I m}(\vec{\xi}) \equiv\left(\begin{array}{c}\mathfrak{I m}\left(\xi_{1}\right) \\ \mathfrak{I m}\left(\xi_{2}\right)\end{array}\right)$. The measure $\mu$ will be the Lebesgue measure on $\mathbb{R}^{2}$, and $L^{2}\left(\mathbb{R}^{2}\right)$ will be the Hilbert space of all square integrable functions on $\mathbb{R}^{2}$. A (countable) set of elements $\left\{\psi_{i}: i \in \Lambda\right\}$ in $L^{2}\left(\mathbb{R}^{2}\right)$ is called a normalized tight frame of $L^{2}\left(\mathbb{R}^{2}\right)$ if

$$
\sum_{i \in \Lambda}\left|\left\langle f, \psi_{i}\right\rangle\right|^{2}=\|f\|^{2}, \quad \forall f \in L^{2}\left(\mathbb{R}^{2}\right) .
$$

It is well known in the literature (see [4]) that the equation (1.1) is equivalent to

$$
f=\sum_{i \in \Lambda}\left\langle f, \psi_{i}\right\rangle \psi_{i}, \quad \forall f \in L^{2}\left(\mathbb{R}^{2}\right) .
$$

Copyright 2016 by the Tusi Mathematical Research Group.

Received Sep. 21, 2015; Accepted Dec. 15, 2015.

2010 Mathematics Subject Classification. Primary 46N99; Secondary 47N99, 46E99, 42C40.

Keywords. Fourier transform, Hilbert space, expansive integral matrix, normalized tight frame wavelet. 


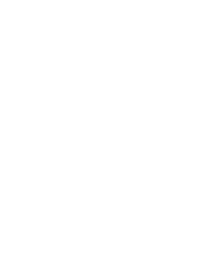

Banach J. Math. Anal. 10 (2016), no. 3, 638-670

http://dx.doi.org/10.1215/17358787-3639712

ISSN: $1735-8787$ (electronic)

http://projecteuclid.org/bjma

\title{
EQUATIONS FOR FRAME WAVELETS IN $L^{2}\left(\mathbb{R}^{2}\right)$
}

\author{
XINGDE DAI \\ To Professor Zurui Guo \\ Communicated by D. Han
}

\begin{abstract}
A finite solution to a system of equations will generate a single function normalized tight frame wavelet (Parseval's frame wavelet) with compact support associated with a $2 \times 2$ expansive integral matrix whose determinant is either 2 or -2 in $L^{2}\left(\mathbb{R}^{2}\right)$.
\end{abstract}

\section{IntRoduction}

In this article, $\mathbb{R}^{2}$ will be the 2-dimensional Euclidean space, and $\mathbb{C}^{2}$ will be the 2-dimensional complex Euclidean space. We will use notation $\vec{t}, \vec{s}, \vec{r}, \vec{\xi}, \vec{\eta}$ for vectors in $\mathbb{R}^{2}$ or $\mathbb{C}^{2}$. We will use the notation $\vec{t}_{1} \circ \vec{t}_{2}$ for the standard inner product of two vectors $\vec{t}_{1}$ and $\vec{t}_{2}$. For a vector $\vec{\xi}=\left(\begin{array}{c}\xi_{1} \\ \xi_{2}\end{array}\right)$ in $\mathbb{C}^{2}$, its real part is $\mathfrak{R e}(\vec{\xi}) \equiv\left(\begin{array}{c}\mathfrak{R e}\left(\xi_{1}\right) \\ \mathfrak{R}\left(\xi_{2}\right)\end{array}\right)$, and its imaginary part is $\mathfrak{I m}(\vec{\xi}) \equiv\left(\begin{array}{c}\mathfrak{I m}\left(\xi_{1}\right) \\ \mathfrak{I m}\left(\xi_{2}\right)\end{array}\right)$. The measure $\mu$ will be the Lebesgue measure on $\mathbb{R}^{2}$, and $L^{2}\left(\mathbb{R}^{2}\right)$ will be the Hilbert space of all square integrable functions on $\mathbb{R}^{2}$. A (countable) set of elements $\left\{\psi_{i}: i \in \Lambda\right\}$ in $L^{2}\left(\mathbb{R}^{2}\right)$ is called a normalized tight frame of $L^{2}\left(\mathbb{R}^{2}\right)$ if

$$
\sum_{i \in \Lambda}\left|\left\langle f, \psi_{i}\right\rangle\right|^{2}=\|f\|^{2}, \quad \forall f \in L^{2}\left(\mathbb{R}^{2}\right) .
$$

It is well known in the literature (see [4]) that the equation (1.1) is equivalent to

$$
f=\sum_{i \in \Lambda}\left\langle f, \psi_{i}\right\rangle \psi_{i}, \quad \forall f \in L^{2}\left(\mathbb{R}^{2}\right) .
$$

Copyright 2016 by the Tusi Mathematical Research Group.

Received Sep. 21, 2015; Accepted Dec. 15, 2015.

2010 Mathematics Subject Classification. Primary 46N99; Secondary 47N99, 46E99, 42C40.

Keywords. Fourier transform, Hilbert space, expansive integral matrix, normalized tight frame wavelet. 


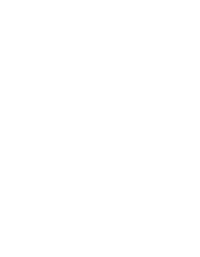

Banach J. Math. Anal. 10 (2016), no. 3, 638-670

http://dx.doi.org/10.1215/17358787-3639712

ISSN: $1735-8787$ (electronic)

http://projecteuclid.org/bjma

\title{
EQUATIONS FOR FRAME WAVELETS IN $L^{2}\left(\mathbb{R}^{2}\right)$
}

\author{
XINGDE DAI \\ To Professor Zurui Guo \\ Communicated by D. Han
}

\begin{abstract}
A finite solution to a system of equations will generate a single function normalized tight frame wavelet (Parseval's frame wavelet) with compact support associated with a $2 \times 2$ expansive integral matrix whose determinant is either 2 or -2 in $L^{2}\left(\mathbb{R}^{2}\right)$.
\end{abstract}

\section{IntRoduction}

In this article, $\mathbb{R}^{2}$ will be the 2-dimensional Euclidean space, and $\mathbb{C}^{2}$ will be the 2-dimensional complex Euclidean space. We will use notation $\vec{t}, \vec{s}, \vec{r}, \vec{\xi}, \vec{\eta}$ for vectors in $\mathbb{R}^{2}$ or $\mathbb{C}^{2}$. We will use the notation $\vec{t}_{1} \circ \vec{t}_{2}$ for the standard inner product of two vectors $\vec{t}_{1}$ and $\vec{t}_{2}$. For a vector $\vec{\xi}=\left(\begin{array}{c}\xi_{1} \\ \xi_{2}\end{array}\right)$ in $\mathbb{C}^{2}$, its real part is $\mathfrak{R e}(\vec{\xi}) \equiv\left(\begin{array}{c}\mathfrak{R e}\left(\xi_{1}\right) \\ \mathfrak{R}\left(\xi_{2}\right)\end{array}\right)$, and its imaginary part is $\mathfrak{I m}(\vec{\xi}) \equiv\left(\begin{array}{c}\mathfrak{I m}\left(\xi_{1}\right) \\ \mathfrak{I m}\left(\xi_{2}\right)\end{array}\right)$. The measure $\mu$ will be the Lebesgue measure on $\mathbb{R}^{2}$, and $L^{2}\left(\mathbb{R}^{2}\right)$ will be the Hilbert space of all square integrable functions on $\mathbb{R}^{2}$. A (countable) set of elements $\left\{\psi_{i}: i \in \Lambda\right\}$ in $L^{2}\left(\mathbb{R}^{2}\right)$ is called a normalized tight frame of $L^{2}\left(\mathbb{R}^{2}\right)$ if

$$
\sum_{i \in \Lambda}\left|\left\langle f, \psi_{i}\right\rangle\right|^{2}=\|f\|^{2}, \quad \forall f \in L^{2}\left(\mathbb{R}^{2}\right) .
$$

It is well known in the literature (see [4]) that the equation (1.1) is equivalent to

$$
f=\sum_{i \in \Lambda}\left\langle f, \psi_{i}\right\rangle \psi_{i}, \quad \forall f \in L^{2}\left(\mathbb{R}^{2}\right) .
$$

Copyright 2016 by the Tusi Mathematical Research Group.

Received Sep. 21, 2015; Accepted Dec. 15, 2015.

2010 Mathematics Subject Classification. Primary 46N99; Secondary 47N99, 46E99, 42C40.

Keywords. Fourier transform, Hilbert space, expansive integral matrix, normalized tight frame wavelet. 


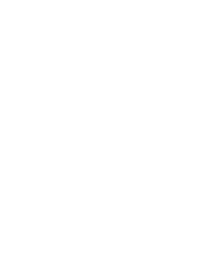

Banach J. Math. Anal. 10 (2016), no. 3, 638-670

http://dx.doi.org/10.1215/17358787-3639712

ISSN: $1735-8787$ (electronic)

http://projecteuclid.org/bjma

\title{
EQUATIONS FOR FRAME WAVELETS IN $L^{2}\left(\mathbb{R}^{2}\right)$
}

\author{
XINGDE DAI \\ To Professor Zurui Guo \\ Communicated by D. Han
}

\begin{abstract}
A finite solution to a system of equations will generate a single function normalized tight frame wavelet (Parseval's frame wavelet) with compact support associated with a $2 \times 2$ expansive integral matrix whose determinant is either 2 or -2 in $L^{2}\left(\mathbb{R}^{2}\right)$.
\end{abstract}

\section{IntRoduction}

In this article, $\mathbb{R}^{2}$ will be the 2-dimensional Euclidean space, and $\mathbb{C}^{2}$ will be the 2-dimensional complex Euclidean space. We will use notation $\vec{t}, \vec{s}, \vec{r}, \vec{\xi}, \vec{\eta}$ for vectors in $\mathbb{R}^{2}$ or $\mathbb{C}^{2}$. We will use the notation $\vec{t}_{1} \circ \vec{t}_{2}$ for the standard inner product of two vectors $\vec{t}_{1}$ and $\vec{t}_{2}$. For a vector $\vec{\xi}=\left(\begin{array}{c}\xi_{1} \\ \xi_{2}\end{array}\right)$ in $\mathbb{C}^{2}$, its real part is $\mathfrak{R e}(\vec{\xi}) \equiv\left(\begin{array}{c}\mathfrak{R e}\left(\xi_{1}\right) \\ \mathfrak{R}\left(\xi_{2}\right)\end{array}\right)$, and its imaginary part is $\mathfrak{I m}(\vec{\xi}) \equiv\left(\begin{array}{c}\mathfrak{I m}\left(\xi_{1}\right) \\ \mathfrak{I m}\left(\xi_{2}\right)\end{array}\right)$. The measure $\mu$ will be the Lebesgue measure on $\mathbb{R}^{2}$, and $L^{2}\left(\mathbb{R}^{2}\right)$ will be the Hilbert space of all square integrable functions on $\mathbb{R}^{2}$. A (countable) set of elements $\left\{\psi_{i}: i \in \Lambda\right\}$ in $L^{2}\left(\mathbb{R}^{2}\right)$ is called a normalized tight frame of $L^{2}\left(\mathbb{R}^{2}\right)$ if

$$
\sum_{i \in \Lambda}\left|\left\langle f, \psi_{i}\right\rangle\right|^{2}=\|f\|^{2}, \quad \forall f \in L^{2}\left(\mathbb{R}^{2}\right) .
$$

It is well known in the literature (see [4]) that the equation (1.1) is equivalent to

$$
f=\sum_{i \in \Lambda}\left\langle f, \psi_{i}\right\rangle \psi_{i}, \quad \forall f \in L^{2}\left(\mathbb{R}^{2}\right) .
$$

Copyright 2016 by the Tusi Mathematical Research Group.

Received Sep. 21, 2015; Accepted Dec. 15, 2015.

2010 Mathematics Subject Classification. Primary 46N99; Secondary 47N99, 46E99, 42C40.

Keywords. Fourier transform, Hilbert space, expansive integral matrix, normalized tight frame wavelet. 


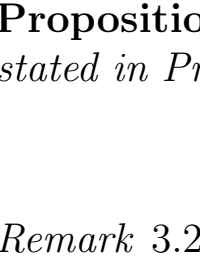

Banach J. Math. Anal. 10 (2016), no. 3, 638-670

http://dx.doi.org/10.1215/17358787-3639712

ISSN: $1735-8787$ (electronic)

http://projecteuclid.org/bjma

\title{
EQUATIONS FOR FRAME WAVELETS IN $L^{2}\left(\mathbb{R}^{2}\right)$
}

\author{
XINGDE DAI \\ To Professor Zurui Guo \\ Communicated by D. Han
}

\begin{abstract}
A finite solution to a system of equations will generate a single function normalized tight frame wavelet (Parseval's frame wavelet) with compact support associated with a $2 \times 2$ expansive integral matrix whose determinant is either 2 or -2 in $L^{2}\left(\mathbb{R}^{2}\right)$.
\end{abstract}

\section{IntRoduction}

In this article, $\mathbb{R}^{2}$ will be the 2-dimensional Euclidean space, and $\mathbb{C}^{2}$ will be the 2-dimensional complex Euclidean space. We will use notation $\vec{t}, \vec{s}, \vec{r}, \vec{\xi}, \vec{\eta}$ for vectors in $\mathbb{R}^{2}$ or $\mathbb{C}^{2}$. We will use the notation $\vec{t}_{1} \circ \vec{t}_{2}$ for the standard inner product of two vectors $\vec{t}_{1}$ and $\vec{t}_{2}$. For a vector $\vec{\xi}=\left(\begin{array}{c}\xi_{1} \\ \xi_{2}\end{array}\right)$ in $\mathbb{C}^{2}$, its real part is $\mathfrak{R e}(\vec{\xi}) \equiv\left(\begin{array}{c}\mathfrak{R e}\left(\xi_{1}\right) \\ \mathfrak{R}\left(\xi_{2}\right)\end{array}\right)$, and its imaginary part is $\mathfrak{I m}(\vec{\xi}) \equiv\left(\begin{array}{c}\mathfrak{I m}\left(\xi_{1}\right) \\ \mathfrak{I m}\left(\xi_{2}\right)\end{array}\right)$. The measure $\mu$ will be the Lebesgue measure on $\mathbb{R}^{2}$, and $L^{2}\left(\mathbb{R}^{2}\right)$ will be the Hilbert space of all square integrable functions on $\mathbb{R}^{2}$. A (countable) set of elements $\left\{\psi_{i}: i \in \Lambda\right\}$ in $L^{2}\left(\mathbb{R}^{2}\right)$ is called a normalized tight frame of $L^{2}\left(\mathbb{R}^{2}\right)$ if

$$
\sum_{i \in \Lambda}\left|\left\langle f, \psi_{i}\right\rangle\right|^{2}=\|f\|^{2}, \quad \forall f \in L^{2}\left(\mathbb{R}^{2}\right) .
$$

It is well known in the literature (see [4]) that the equation (1.1) is equivalent to

$$
f=\sum_{i \in \Lambda}\left\langle f, \psi_{i}\right\rangle \psi_{i}, \quad \forall f \in L^{2}\left(\mathbb{R}^{2}\right) .
$$

Copyright 2016 by the Tusi Mathematical Research Group.

Received Sep. 21, 2015; Accepted Dec. 15, 2015.

2010 Mathematics Subject Classification. Primary 46N99; Secondary 47N99, 46E99, 42C40.

Keywords. Fourier transform, Hilbert space, expansive integral matrix, normalized tight frame wavelet. 


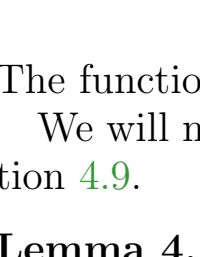

Banach J. Math. Anal. 10 (2016), no. 3, 638-670

http://dx.doi.org/10.1215/17358787-3639712

ISSN: $1735-8787$ (electronic)

http://projecteuclid.org/bjma

\title{
EQUATIONS FOR FRAME WAVELETS IN $L^{2}\left(\mathbb{R}^{2}\right)$
}

\author{
XINGDE DAI \\ To Professor Zurui Guo \\ Communicated by D. Han
}

\begin{abstract}
A finite solution to a system of equations will generate a single function normalized tight frame wavelet (Parseval's frame wavelet) with compact support associated with a $2 \times 2$ expansive integral matrix whose determinant is either 2 or -2 in $L^{2}\left(\mathbb{R}^{2}\right)$.
\end{abstract}

\section{IntRoduction}

In this article, $\mathbb{R}^{2}$ will be the 2-dimensional Euclidean space, and $\mathbb{C}^{2}$ will be the 2-dimensional complex Euclidean space. We will use notation $\vec{t}, \vec{s}, \vec{r}, \vec{\xi}, \vec{\eta}$ for vectors in $\mathbb{R}^{2}$ or $\mathbb{C}^{2}$. We will use the notation $\vec{t}_{1} \circ \vec{t}_{2}$ for the standard inner product of two vectors $\vec{t}_{1}$ and $\vec{t}_{2}$. For a vector $\vec{\xi}=\left(\begin{array}{c}\xi_{1} \\ \xi_{2}\end{array}\right)$ in $\mathbb{C}^{2}$, its real part is $\mathfrak{R e}(\vec{\xi}) \equiv\left(\begin{array}{c}\mathfrak{R e}\left(\xi_{1}\right) \\ \mathfrak{R}\left(\xi_{2}\right)\end{array}\right)$, and its imaginary part is $\mathfrak{I m}(\vec{\xi}) \equiv\left(\begin{array}{c}\mathfrak{I m}\left(\xi_{1}\right) \\ \mathfrak{I m}\left(\xi_{2}\right)\end{array}\right)$. The measure $\mu$ will be the Lebesgue measure on $\mathbb{R}^{2}$, and $L^{2}\left(\mathbb{R}^{2}\right)$ will be the Hilbert space of all square integrable functions on $\mathbb{R}^{2}$. A (countable) set of elements $\left\{\psi_{i}: i \in \Lambda\right\}$ in $L^{2}\left(\mathbb{R}^{2}\right)$ is called a normalized tight frame of $L^{2}\left(\mathbb{R}^{2}\right)$ if

$$
\sum_{i \in \Lambda}\left|\left\langle f, \psi_{i}\right\rangle\right|^{2}=\|f\|^{2}, \quad \forall f \in L^{2}\left(\mathbb{R}^{2}\right) .
$$

It is well known in the literature (see [4]) that the equation (1.1) is equivalent to

$$
f=\sum_{i \in \Lambda}\left\langle f, \psi_{i}\right\rangle \psi_{i}, \quad \forall f \in L^{2}\left(\mathbb{R}^{2}\right) .
$$

Copyright 2016 by the Tusi Mathematical Research Group.

Received Sep. 21, 2015; Accepted Dec. 15, 2015.

2010 Mathematics Subject Classification. Primary 46N99; Secondary 47N99, 46E99, 42C40.

Keywords. Fourier transform, Hilbert space, expansive integral matrix, normalized tight frame wavelet. 


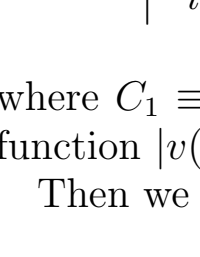

Banach J. Math. Anal. 10 (2016), no. 3, 638-670

http://dx.doi.org/10.1215/17358787-3639712

ISSN: $1735-8787$ (electronic)

http://projecteuclid.org/bjma

\title{
EQUATIONS FOR FRAME WAVELETS IN $L^{2}\left(\mathbb{R}^{2}\right)$
}

\author{
XINGDE DAI \\ To Professor Zurui Guo \\ Communicated by D. Han
}

\begin{abstract}
A finite solution to a system of equations will generate a single function normalized tight frame wavelet (Parseval's frame wavelet) with compact support associated with a $2 \times 2$ expansive integral matrix whose determinant is either 2 or -2 in $L^{2}\left(\mathbb{R}^{2}\right)$.
\end{abstract}

\section{IntRoduction}

In this article, $\mathbb{R}^{2}$ will be the 2-dimensional Euclidean space, and $\mathbb{C}^{2}$ will be the 2-dimensional complex Euclidean space. We will use notation $\vec{t}, \vec{s}, \vec{r}, \vec{\xi}, \vec{\eta}$ for vectors in $\mathbb{R}^{2}$ or $\mathbb{C}^{2}$. We will use the notation $\vec{t}_{1} \circ \vec{t}_{2}$ for the standard inner product of two vectors $\vec{t}_{1}$ and $\vec{t}_{2}$. For a vector $\vec{\xi}=\left(\begin{array}{c}\xi_{1} \\ \xi_{2}\end{array}\right)$ in $\mathbb{C}^{2}$, its real part is $\mathfrak{R e}(\vec{\xi}) \equiv\left(\begin{array}{c}\mathfrak{R e}\left(\xi_{1}\right) \\ \mathfrak{R}\left(\xi_{2}\right)\end{array}\right)$, and its imaginary part is $\mathfrak{I m}(\vec{\xi}) \equiv\left(\begin{array}{c}\mathfrak{I m}\left(\xi_{1}\right) \\ \mathfrak{I m}\left(\xi_{2}\right)\end{array}\right)$. The measure $\mu$ will be the Lebesgue measure on $\mathbb{R}^{2}$, and $L^{2}\left(\mathbb{R}^{2}\right)$ will be the Hilbert space of all square integrable functions on $\mathbb{R}^{2}$. A (countable) set of elements $\left\{\psi_{i}: i \in \Lambda\right\}$ in $L^{2}\left(\mathbb{R}^{2}\right)$ is called a normalized tight frame of $L^{2}\left(\mathbb{R}^{2}\right)$ if

$$
\sum_{i \in \Lambda}\left|\left\langle f, \psi_{i}\right\rangle\right|^{2}=\|f\|^{2}, \quad \forall f \in L^{2}\left(\mathbb{R}^{2}\right) .
$$

It is well known in the literature (see [4]) that the equation (1.1) is equivalent to

$$
f=\sum_{i \in \Lambda}\left\langle f, \psi_{i}\right\rangle \psi_{i}, \quad \forall f \in L^{2}\left(\mathbb{R}^{2}\right) .
$$

Copyright 2016 by the Tusi Mathematical Research Group.

Received Sep. 21, 2015; Accepted Dec. 15, 2015.

2010 Mathematics Subject Classification. Primary 46N99; Secondary 47N99, 46E99, 42C40.

Keywords. Fourier transform, Hilbert space, expansive integral matrix, normalized tight frame wavelet. 


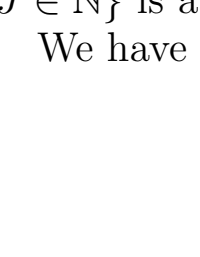

Banach J. Math. Anal. 10 (2016), no. 3, 638-670

http://dx.doi.org/10.1215/17358787-3639712

ISSN: $1735-8787$ (electronic)

http://projecteuclid.org/bjma

\title{
EQUATIONS FOR FRAME WAVELETS IN $L^{2}\left(\mathbb{R}^{2}\right)$
}

\author{
XINGDE DAI \\ To Professor Zurui Guo \\ Communicated by D. Han
}

\begin{abstract}
A finite solution to a system of equations will generate a single function normalized tight frame wavelet (Parseval's frame wavelet) with compact support associated with a $2 \times 2$ expansive integral matrix whose determinant is either 2 or -2 in $L^{2}\left(\mathbb{R}^{2}\right)$.
\end{abstract}

\section{IntRoduction}

In this article, $\mathbb{R}^{2}$ will be the 2-dimensional Euclidean space, and $\mathbb{C}^{2}$ will be the 2-dimensional complex Euclidean space. We will use notation $\vec{t}, \vec{s}, \vec{r}, \vec{\xi}, \vec{\eta}$ for vectors in $\mathbb{R}^{2}$ or $\mathbb{C}^{2}$. We will use the notation $\vec{t}_{1} \circ \vec{t}_{2}$ for the standard inner product of two vectors $\vec{t}_{1}$ and $\vec{t}_{2}$. For a vector $\vec{\xi}=\left(\begin{array}{c}\xi_{1} \\ \xi_{2}\end{array}\right)$ in $\mathbb{C}^{2}$, its real part is $\mathfrak{R e}(\vec{\xi}) \equiv\left(\begin{array}{c}\mathfrak{R e}\left(\xi_{1}\right) \\ \mathfrak{R}\left(\xi_{2}\right)\end{array}\right)$, and its imaginary part is $\mathfrak{I m}(\vec{\xi}) \equiv\left(\begin{array}{c}\mathfrak{I m}\left(\xi_{1}\right) \\ \mathfrak{I m}\left(\xi_{2}\right)\end{array}\right)$. The measure $\mu$ will be the Lebesgue measure on $\mathbb{R}^{2}$, and $L^{2}\left(\mathbb{R}^{2}\right)$ will be the Hilbert space of all square integrable functions on $\mathbb{R}^{2}$. A (countable) set of elements $\left\{\psi_{i}: i \in \Lambda\right\}$ in $L^{2}\left(\mathbb{R}^{2}\right)$ is called a normalized tight frame of $L^{2}\left(\mathbb{R}^{2}\right)$ if

$$
\sum_{i \in \Lambda}\left|\left\langle f, \psi_{i}\right\rangle\right|^{2}=\|f\|^{2}, \quad \forall f \in L^{2}\left(\mathbb{R}^{2}\right) .
$$

It is well known in the literature (see [4]) that the equation (1.1) is equivalent to

$$
f=\sum_{i \in \Lambda}\left\langle f, \psi_{i}\right\rangle \psi_{i}, \quad \forall f \in L^{2}\left(\mathbb{R}^{2}\right) .
$$

Copyright 2016 by the Tusi Mathematical Research Group.

Received Sep. 21, 2015; Accepted Dec. 15, 2015.

2010 Mathematics Subject Classification. Primary 46N99; Secondary 47N99, 46E99, 42C40.

Keywords. Fourier transform, Hilbert space, expansive integral matrix, normalized tight frame wavelet. 


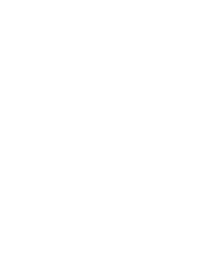

Banach J. Math. Anal. 10 (2016), no. 3, 638-670

http://dx.doi.org/10.1215/17358787-3639712

ISSN: $1735-8787$ (electronic)

http://projecteuclid.org/bjma

\title{
EQUATIONS FOR FRAME WAVELETS IN $L^{2}\left(\mathbb{R}^{2}\right)$
}

\author{
XINGDE DAI \\ To Professor Zurui Guo \\ Communicated by D. Han
}

\begin{abstract}
A finite solution to a system of equations will generate a single function normalized tight frame wavelet (Parseval's frame wavelet) with compact support associated with a $2 \times 2$ expansive integral matrix whose determinant is either 2 or -2 in $L^{2}\left(\mathbb{R}^{2}\right)$.
\end{abstract}

\section{IntRoduction}

In this article, $\mathbb{R}^{2}$ will be the 2-dimensional Euclidean space, and $\mathbb{C}^{2}$ will be the 2-dimensional complex Euclidean space. We will use notation $\vec{t}, \vec{s}, \vec{r}, \vec{\xi}, \vec{\eta}$ for vectors in $\mathbb{R}^{2}$ or $\mathbb{C}^{2}$. We will use the notation $\vec{t}_{1} \circ \vec{t}_{2}$ for the standard inner product of two vectors $\vec{t}_{1}$ and $\vec{t}_{2}$. For a vector $\vec{\xi}=\left(\begin{array}{c}\xi_{1} \\ \xi_{2}\end{array}\right)$ in $\mathbb{C}^{2}$, its real part is $\mathfrak{R e}(\vec{\xi}) \equiv\left(\begin{array}{c}\mathfrak{R e}\left(\xi_{1}\right) \\ \mathfrak{R}\left(\xi_{2}\right)\end{array}\right)$, and its imaginary part is $\mathfrak{I m}(\vec{\xi}) \equiv\left(\begin{array}{c}\mathfrak{I m}\left(\xi_{1}\right) \\ \mathfrak{I m}\left(\xi_{2}\right)\end{array}\right)$. The measure $\mu$ will be the Lebesgue measure on $\mathbb{R}^{2}$, and $L^{2}\left(\mathbb{R}^{2}\right)$ will be the Hilbert space of all square integrable functions on $\mathbb{R}^{2}$. A (countable) set of elements $\left\{\psi_{i}: i \in \Lambda\right\}$ in $L^{2}\left(\mathbb{R}^{2}\right)$ is called a normalized tight frame of $L^{2}\left(\mathbb{R}^{2}\right)$ if

$$
\sum_{i \in \Lambda}\left|\left\langle f, \psi_{i}\right\rangle\right|^{2}=\|f\|^{2}, \quad \forall f \in L^{2}\left(\mathbb{R}^{2}\right) .
$$

It is well known in the literature (see [4]) that the equation (1.1) is equivalent to

$$
f=\sum_{i \in \Lambda}\left\langle f, \psi_{i}\right\rangle \psi_{i}, \quad \forall f \in L^{2}\left(\mathbb{R}^{2}\right) .
$$

Copyright 2016 by the Tusi Mathematical Research Group.

Received Sep. 21, 2015; Accepted Dec. 15, 2015.

2010 Mathematics Subject Classification. Primary 46N99; Secondary 47N99, 46E99, 42C40.

Keywords. Fourier transform, Hilbert space, expansive integral matrix, normalized tight frame wavelet. 


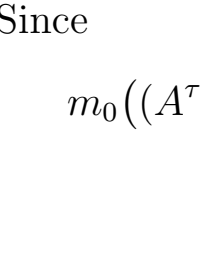

Banach J. Math. Anal. 10 (2016), no. 3, 638-670

http://dx.doi.org/10.1215/17358787-3639712

ISSN: $1735-8787$ (electronic)

http://projecteuclid.org/bjma

\title{
EQUATIONS FOR FRAME WAVELETS IN $L^{2}\left(\mathbb{R}^{2}\right)$
}

\author{
XINGDE DAI \\ To Professor Zurui Guo \\ Communicated by D. Han
}

\begin{abstract}
A finite solution to a system of equations will generate a single function normalized tight frame wavelet (Parseval's frame wavelet) with compact support associated with a $2 \times 2$ expansive integral matrix whose determinant is either 2 or -2 in $L^{2}\left(\mathbb{R}^{2}\right)$.
\end{abstract}

\section{IntRoduction}

In this article, $\mathbb{R}^{2}$ will be the 2-dimensional Euclidean space, and $\mathbb{C}^{2}$ will be the 2-dimensional complex Euclidean space. We will use notation $\vec{t}, \vec{s}, \vec{r}, \vec{\xi}, \vec{\eta}$ for vectors in $\mathbb{R}^{2}$ or $\mathbb{C}^{2}$. We will use the notation $\vec{t}_{1} \circ \vec{t}_{2}$ for the standard inner product of two vectors $\vec{t}_{1}$ and $\vec{t}_{2}$. For a vector $\vec{\xi}=\left(\begin{array}{c}\xi_{1} \\ \xi_{2}\end{array}\right)$ in $\mathbb{C}^{2}$, its real part is $\mathfrak{R e}(\vec{\xi}) \equiv\left(\begin{array}{c}\mathfrak{R e}\left(\xi_{1}\right) \\ \mathfrak{R}\left(\xi_{2}\right)\end{array}\right)$, and its imaginary part is $\mathfrak{I m}(\vec{\xi}) \equiv\left(\begin{array}{c}\mathfrak{I m}\left(\xi_{1}\right) \\ \mathfrak{I m}\left(\xi_{2}\right)\end{array}\right)$. The measure $\mu$ will be the Lebesgue measure on $\mathbb{R}^{2}$, and $L^{2}\left(\mathbb{R}^{2}\right)$ will be the Hilbert space of all square integrable functions on $\mathbb{R}^{2}$. A (countable) set of elements $\left\{\psi_{i}: i \in \Lambda\right\}$ in $L^{2}\left(\mathbb{R}^{2}\right)$ is called a normalized tight frame of $L^{2}\left(\mathbb{R}^{2}\right)$ if

$$
\sum_{i \in \Lambda}\left|\left\langle f, \psi_{i}\right\rangle\right|^{2}=\|f\|^{2}, \quad \forall f \in L^{2}\left(\mathbb{R}^{2}\right) .
$$

It is well known in the literature (see [4]) that the equation (1.1) is equivalent to

$$
f=\sum_{i \in \Lambda}\left\langle f, \psi_{i}\right\rangle \psi_{i}, \quad \forall f \in L^{2}\left(\mathbb{R}^{2}\right) .
$$

Copyright 2016 by the Tusi Mathematical Research Group.

Received Sep. 21, 2015; Accepted Dec. 15, 2015.

2010 Mathematics Subject Classification. Primary 46N99; Secondary 47N99, 46E99, 42C40.

Keywords. Fourier transform, Hilbert space, expansive integral matrix, normalized tight frame wavelet. 


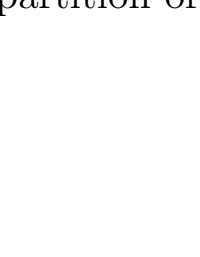

Banach J. Math. Anal. 10 (2016), no. 3, 638-670

http://dx.doi.org/10.1215/17358787-3639712

ISSN: $1735-8787$ (electronic)

http://projecteuclid.org/bjma

\title{
EQUATIONS FOR FRAME WAVELETS IN $L^{2}\left(\mathbb{R}^{2}\right)$
}

\author{
XINGDE DAI \\ To Professor Zurui Guo \\ Communicated by D. Han
}

\begin{abstract}
A finite solution to a system of equations will generate a single function normalized tight frame wavelet (Parseval's frame wavelet) with compact support associated with a $2 \times 2$ expansive integral matrix whose determinant is either 2 or -2 in $L^{2}\left(\mathbb{R}^{2}\right)$.
\end{abstract}

\section{IntRoduction}

In this article, $\mathbb{R}^{2}$ will be the 2-dimensional Euclidean space, and $\mathbb{C}^{2}$ will be the 2-dimensional complex Euclidean space. We will use notation $\vec{t}, \vec{s}, \vec{r}, \vec{\xi}, \vec{\eta}$ for vectors in $\mathbb{R}^{2}$ or $\mathbb{C}^{2}$. We will use the notation $\vec{t}_{1} \circ \vec{t}_{2}$ for the standard inner product of two vectors $\vec{t}_{1}$ and $\vec{t}_{2}$. For a vector $\vec{\xi}=\left(\begin{array}{c}\xi_{1} \\ \xi_{2}\end{array}\right)$ in $\mathbb{C}^{2}$, its real part is $\mathfrak{R e}(\vec{\xi}) \equiv\left(\begin{array}{c}\mathfrak{R e}\left(\xi_{1}\right) \\ \mathfrak{R}\left(\xi_{2}\right)\end{array}\right)$, and its imaginary part is $\mathfrak{I m}(\vec{\xi}) \equiv\left(\begin{array}{c}\mathfrak{I m}\left(\xi_{1}\right) \\ \mathfrak{I m}\left(\xi_{2}\right)\end{array}\right)$. The measure $\mu$ will be the Lebesgue measure on $\mathbb{R}^{2}$, and $L^{2}\left(\mathbb{R}^{2}\right)$ will be the Hilbert space of all square integrable functions on $\mathbb{R}^{2}$. A (countable) set of elements $\left\{\psi_{i}: i \in \Lambda\right\}$ in $L^{2}\left(\mathbb{R}^{2}\right)$ is called a normalized tight frame of $L^{2}\left(\mathbb{R}^{2}\right)$ if

$$
\sum_{i \in \Lambda}\left|\left\langle f, \psi_{i}\right\rangle\right|^{2}=\|f\|^{2}, \quad \forall f \in L^{2}\left(\mathbb{R}^{2}\right) .
$$

It is well known in the literature (see [4]) that the equation (1.1) is equivalent to

$$
f=\sum_{i \in \Lambda}\left\langle f, \psi_{i}\right\rangle \psi_{i}, \quad \forall f \in L^{2}\left(\mathbb{R}^{2}\right) .
$$

Copyright 2016 by the Tusi Mathematical Research Group.

Received Sep. 21, 2015; Accepted Dec. 15, 2015.

2010 Mathematics Subject Classification. Primary 46N99; Secondary 47N99, 46E99, 42C40.

Keywords. Fourier transform, Hilbert space, expansive integral matrix, normalized tight frame wavelet. 


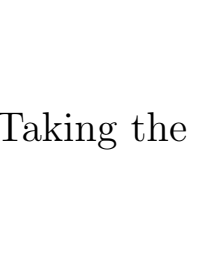

Banach J. Math. Anal. 10 (2016), no. 3, 638-670

http://dx.doi.org/10.1215/17358787-3639712

ISSN: $1735-8787$ (electronic)

http://projecteuclid.org/bjma

\title{
EQUATIONS FOR FRAME WAVELETS IN $L^{2}\left(\mathbb{R}^{2}\right)$
}

\author{
XINGDE DAI \\ To Professor Zurui Guo \\ Communicated by D. Han
}

\begin{abstract}
A finite solution to a system of equations will generate a single function normalized tight frame wavelet (Parseval's frame wavelet) with compact support associated with a $2 \times 2$ expansive integral matrix whose determinant is either 2 or -2 in $L^{2}\left(\mathbb{R}^{2}\right)$.
\end{abstract}

\section{IntRoduction}

In this article, $\mathbb{R}^{2}$ will be the 2-dimensional Euclidean space, and $\mathbb{C}^{2}$ will be the 2-dimensional complex Euclidean space. We will use notation $\vec{t}, \vec{s}, \vec{r}, \vec{\xi}, \vec{\eta}$ for vectors in $\mathbb{R}^{2}$ or $\mathbb{C}^{2}$. We will use the notation $\vec{t}_{1} \circ \vec{t}_{2}$ for the standard inner product of two vectors $\vec{t}_{1}$ and $\vec{t}_{2}$. For a vector $\vec{\xi}=\left(\begin{array}{c}\xi_{1} \\ \xi_{2}\end{array}\right)$ in $\mathbb{C}^{2}$, its real part is $\mathfrak{R e}(\vec{\xi}) \equiv\left(\begin{array}{c}\mathfrak{R e}\left(\xi_{1}\right) \\ \mathfrak{R}\left(\xi_{2}\right)\end{array}\right)$, and its imaginary part is $\mathfrak{I m}(\vec{\xi}) \equiv\left(\begin{array}{c}\mathfrak{I m}\left(\xi_{1}\right) \\ \mathfrak{I m}\left(\xi_{2}\right)\end{array}\right)$. The measure $\mu$ will be the Lebesgue measure on $\mathbb{R}^{2}$, and $L^{2}\left(\mathbb{R}^{2}\right)$ will be the Hilbert space of all square integrable functions on $\mathbb{R}^{2}$. A (countable) set of elements $\left\{\psi_{i}: i \in \Lambda\right\}$ in $L^{2}\left(\mathbb{R}^{2}\right)$ is called a normalized tight frame of $L^{2}\left(\mathbb{R}^{2}\right)$ if

$$
\sum_{i \in \Lambda}\left|\left\langle f, \psi_{i}\right\rangle\right|^{2}=\|f\|^{2}, \quad \forall f \in L^{2}\left(\mathbb{R}^{2}\right) .
$$

It is well known in the literature (see [4]) that the equation (1.1) is equivalent to

$$
f=\sum_{i \in \Lambda}\left\langle f, \psi_{i}\right\rangle \psi_{i}, \quad \forall f \in L^{2}\left(\mathbb{R}^{2}\right) .
$$

Copyright 2016 by the Tusi Mathematical Research Group.

Received Sep. 21, 2015; Accepted Dec. 15, 2015.

2010 Mathematics Subject Classification. Primary 46N99; Secondary 47N99, 46E99, 42C40.

Keywords. Fourier transform, Hilbert space, expansive integral matrix, normalized tight frame wavelet. 


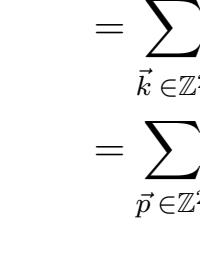

Banach J. Math. Anal. 10 (2016), no. 3, 638-670

http://dx.doi.org/10.1215/17358787-3639712

ISSN: $1735-8787$ (electronic)

http://projecteuclid.org/bjma

\title{
EQUATIONS FOR FRAME WAVELETS IN $L^{2}\left(\mathbb{R}^{2}\right)$
}

\author{
XINGDE DAI \\ To Professor Zurui Guo \\ Communicated by D. Han
}

\begin{abstract}
A finite solution to a system of equations will generate a single function normalized tight frame wavelet (Parseval's frame wavelet) with compact support associated with a $2 \times 2$ expansive integral matrix whose determinant is either 2 or -2 in $L^{2}\left(\mathbb{R}^{2}\right)$.
\end{abstract}

\section{IntRoduction}

In this article, $\mathbb{R}^{2}$ will be the 2-dimensional Euclidean space, and $\mathbb{C}^{2}$ will be the 2-dimensional complex Euclidean space. We will use notation $\vec{t}, \vec{s}, \vec{r}, \vec{\xi}, \vec{\eta}$ for vectors in $\mathbb{R}^{2}$ or $\mathbb{C}^{2}$. We will use the notation $\vec{t}_{1} \circ \vec{t}_{2}$ for the standard inner product of two vectors $\vec{t}_{1}$ and $\vec{t}_{2}$. For a vector $\vec{\xi}=\left(\begin{array}{c}\xi_{1} \\ \xi_{2}\end{array}\right)$ in $\mathbb{C}^{2}$, its real part is $\mathfrak{R e}(\vec{\xi}) \equiv\left(\begin{array}{c}\mathfrak{R e}\left(\xi_{1}\right) \\ \mathfrak{R}\left(\xi_{2}\right)\end{array}\right)$, and its imaginary part is $\mathfrak{I m}(\vec{\xi}) \equiv\left(\begin{array}{c}\mathfrak{I m}\left(\xi_{1}\right) \\ \mathfrak{I m}\left(\xi_{2}\right)\end{array}\right)$. The measure $\mu$ will be the Lebesgue measure on $\mathbb{R}^{2}$, and $L^{2}\left(\mathbb{R}^{2}\right)$ will be the Hilbert space of all square integrable functions on $\mathbb{R}^{2}$. A (countable) set of elements $\left\{\psi_{i}: i \in \Lambda\right\}$ in $L^{2}\left(\mathbb{R}^{2}\right)$ is called a normalized tight frame of $L^{2}\left(\mathbb{R}^{2}\right)$ if

$$
\sum_{i \in \Lambda}\left|\left\langle f, \psi_{i}\right\rangle\right|^{2}=\|f\|^{2}, \quad \forall f \in L^{2}\left(\mathbb{R}^{2}\right) .
$$

It is well known in the literature (see [4]) that the equation (1.1) is equivalent to

$$
f=\sum_{i \in \Lambda}\left\langle f, \psi_{i}\right\rangle \psi_{i}, \quad \forall f \in L^{2}\left(\mathbb{R}^{2}\right) .
$$

Copyright 2016 by the Tusi Mathematical Research Group.

Received Sep. 21, 2015; Accepted Dec. 15, 2015.

2010 Mathematics Subject Classification. Primary 46N99; Secondary 47N99, 46E99, 42C40.

Keywords. Fourier transform, Hilbert space, expansive integral matrix, normalized tight frame wavelet. 


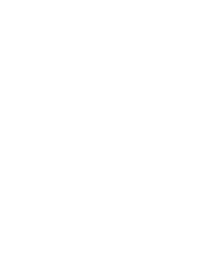

Banach J. Math. Anal. 10 (2016), no. 3, 638-670

http://dx.doi.org/10.1215/17358787-3639712

ISSN: $1735-8787$ (electronic)

http://projecteuclid.org/bjma

\title{
EQUATIONS FOR FRAME WAVELETS IN $L^{2}\left(\mathbb{R}^{2}\right)$
}

\author{
XINGDE DAI \\ To Professor Zurui Guo \\ Communicated by D. Han
}

\begin{abstract}
A finite solution to a system of equations will generate a single function normalized tight frame wavelet (Parseval's frame wavelet) with compact support associated with a $2 \times 2$ expansive integral matrix whose determinant is either 2 or -2 in $L^{2}\left(\mathbb{R}^{2}\right)$.
\end{abstract}

\section{IntRoduction}

In this article, $\mathbb{R}^{2}$ will be the 2-dimensional Euclidean space, and $\mathbb{C}^{2}$ will be the 2-dimensional complex Euclidean space. We will use notation $\vec{t}, \vec{s}, \vec{r}, \vec{\xi}, \vec{\eta}$ for vectors in $\mathbb{R}^{2}$ or $\mathbb{C}^{2}$. We will use the notation $\vec{t}_{1} \circ \vec{t}_{2}$ for the standard inner product of two vectors $\vec{t}_{1}$ and $\vec{t}_{2}$. For a vector $\vec{\xi}=\left(\begin{array}{c}\xi_{1} \\ \xi_{2}\end{array}\right)$ in $\mathbb{C}^{2}$, its real part is $\mathfrak{R e}(\vec{\xi}) \equiv\left(\begin{array}{c}\mathfrak{R e}\left(\xi_{1}\right) \\ \mathfrak{R}\left(\xi_{2}\right)\end{array}\right)$, and its imaginary part is $\mathfrak{I m}(\vec{\xi}) \equiv\left(\begin{array}{c}\mathfrak{I m}\left(\xi_{1}\right) \\ \mathfrak{I m}\left(\xi_{2}\right)\end{array}\right)$. The measure $\mu$ will be the Lebesgue measure on $\mathbb{R}^{2}$, and $L^{2}\left(\mathbb{R}^{2}\right)$ will be the Hilbert space of all square integrable functions on $\mathbb{R}^{2}$. A (countable) set of elements $\left\{\psi_{i}: i \in \Lambda\right\}$ in $L^{2}\left(\mathbb{R}^{2}\right)$ is called a normalized tight frame of $L^{2}\left(\mathbb{R}^{2}\right)$ if

$$
\sum_{i \in \Lambda}\left|\left\langle f, \psi_{i}\right\rangle\right|^{2}=\|f\|^{2}, \quad \forall f \in L^{2}\left(\mathbb{R}^{2}\right) .
$$

It is well known in the literature (see [4]) that the equation (1.1) is equivalent to

$$
f=\sum_{i \in \Lambda}\left\langle f, \psi_{i}\right\rangle \psi_{i}, \quad \forall f \in L^{2}\left(\mathbb{R}^{2}\right) .
$$

Copyright 2016 by the Tusi Mathematical Research Group.

Received Sep. 21, 2015; Accepted Dec. 15, 2015.

2010 Mathematics Subject Classification. Primary 46N99; Secondary 47N99, 46E99, 42C40.

Keywords. Fourier transform, Hilbert space, expansive integral matrix, normalized tight frame wavelet. 


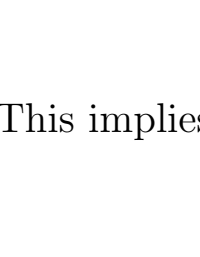

Banach J. Math. Anal. 10 (2016), no. 3, 638-670

http://dx.doi.org/10.1215/17358787-3639712

ISSN: $1735-8787$ (electronic)

http://projecteuclid.org/bjma

\title{
EQUATIONS FOR FRAME WAVELETS IN $L^{2}\left(\mathbb{R}^{2}\right)$
}

\author{
XINGDE DAI \\ To Professor Zurui Guo \\ Communicated by D. Han
}

\begin{abstract}
A finite solution to a system of equations will generate a single function normalized tight frame wavelet (Parseval's frame wavelet) with compact support associated with a $2 \times 2$ expansive integral matrix whose determinant is either 2 or -2 in $L^{2}\left(\mathbb{R}^{2}\right)$.
\end{abstract}

\section{IntRoduction}

In this article, $\mathbb{R}^{2}$ will be the 2-dimensional Euclidean space, and $\mathbb{C}^{2}$ will be the 2-dimensional complex Euclidean space. We will use notation $\vec{t}, \vec{s}, \vec{r}, \vec{\xi}, \vec{\eta}$ for vectors in $\mathbb{R}^{2}$ or $\mathbb{C}^{2}$. We will use the notation $\vec{t}_{1} \circ \vec{t}_{2}$ for the standard inner product of two vectors $\vec{t}_{1}$ and $\vec{t}_{2}$. For a vector $\vec{\xi}=\left(\begin{array}{c}\xi_{1} \\ \xi_{2}\end{array}\right)$ in $\mathbb{C}^{2}$, its real part is $\mathfrak{R e}(\vec{\xi}) \equiv\left(\begin{array}{c}\mathfrak{R e}\left(\xi_{1}\right) \\ \mathfrak{R}\left(\xi_{2}\right)\end{array}\right)$, and its imaginary part is $\mathfrak{I m}(\vec{\xi}) \equiv\left(\begin{array}{c}\mathfrak{I m}\left(\xi_{1}\right) \\ \mathfrak{I m}\left(\xi_{2}\right)\end{array}\right)$. The measure $\mu$ will be the Lebesgue measure on $\mathbb{R}^{2}$, and $L^{2}\left(\mathbb{R}^{2}\right)$ will be the Hilbert space of all square integrable functions on $\mathbb{R}^{2}$. A (countable) set of elements $\left\{\psi_{i}: i \in \Lambda\right\}$ in $L^{2}\left(\mathbb{R}^{2}\right)$ is called a normalized tight frame of $L^{2}\left(\mathbb{R}^{2}\right)$ if

$$
\sum_{i \in \Lambda}\left|\left\langle f, \psi_{i}\right\rangle\right|^{2}=\|f\|^{2}, \quad \forall f \in L^{2}\left(\mathbb{R}^{2}\right) .
$$

It is well known in the literature (see [4]) that the equation (1.1) is equivalent to

$$
f=\sum_{i \in \Lambda}\left\langle f, \psi_{i}\right\rangle \psi_{i}, \quad \forall f \in L^{2}\left(\mathbb{R}^{2}\right) .
$$

Copyright 2016 by the Tusi Mathematical Research Group.

Received Sep. 21, 2015; Accepted Dec. 15, 2015.

2010 Mathematics Subject Classification. Primary 46N99; Secondary 47N99, 46E99, 42C40.

Keywords. Fourier transform, Hilbert space, expansive integral matrix, normalized tight frame wavelet. 


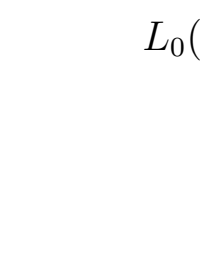

Banach J. Math. Anal. 10 (2016), no. 3, 638-670

http://dx.doi.org/10.1215/17358787-3639712

ISSN: $1735-8787$ (electronic)

http://projecteuclid.org/bjma

\title{
EQUATIONS FOR FRAME WAVELETS IN $L^{2}\left(\mathbb{R}^{2}\right)$
}

\author{
XINGDE DAI \\ To Professor Zurui Guo \\ Communicated by D. Han
}

\begin{abstract}
A finite solution to a system of equations will generate a single function normalized tight frame wavelet (Parseval's frame wavelet) with compact support associated with a $2 \times 2$ expansive integral matrix whose determinant is either 2 or -2 in $L^{2}\left(\mathbb{R}^{2}\right)$.
\end{abstract}

\section{IntRoduction}

In this article, $\mathbb{R}^{2}$ will be the 2-dimensional Euclidean space, and $\mathbb{C}^{2}$ will be the 2-dimensional complex Euclidean space. We will use notation $\vec{t}, \vec{s}, \vec{r}, \vec{\xi}, \vec{\eta}$ for vectors in $\mathbb{R}^{2}$ or $\mathbb{C}^{2}$. We will use the notation $\vec{t}_{1} \circ \vec{t}_{2}$ for the standard inner product of two vectors $\vec{t}_{1}$ and $\vec{t}_{2}$. For a vector $\vec{\xi}=\left(\begin{array}{c}\xi_{1} \\ \xi_{2}\end{array}\right)$ in $\mathbb{C}^{2}$, its real part is $\mathfrak{R e}(\vec{\xi}) \equiv\left(\begin{array}{c}\mathfrak{R e}\left(\xi_{1}\right) \\ \mathfrak{R}\left(\xi_{2}\right)\end{array}\right)$, and its imaginary part is $\mathfrak{I m}(\vec{\xi}) \equiv\left(\begin{array}{c}\mathfrak{I m}\left(\xi_{1}\right) \\ \mathfrak{I m}\left(\xi_{2}\right)\end{array}\right)$. The measure $\mu$ will be the Lebesgue measure on $\mathbb{R}^{2}$, and $L^{2}\left(\mathbb{R}^{2}\right)$ will be the Hilbert space of all square integrable functions on $\mathbb{R}^{2}$. A (countable) set of elements $\left\{\psi_{i}: i \in \Lambda\right\}$ in $L^{2}\left(\mathbb{R}^{2}\right)$ is called a normalized tight frame of $L^{2}\left(\mathbb{R}^{2}\right)$ if

$$
\sum_{i \in \Lambda}\left|\left\langle f, \psi_{i}\right\rangle\right|^{2}=\|f\|^{2}, \quad \forall f \in L^{2}\left(\mathbb{R}^{2}\right) .
$$

It is well known in the literature (see [4]) that the equation (1.1) is equivalent to

$$
f=\sum_{i \in \Lambda}\left\langle f, \psi_{i}\right\rangle \psi_{i}, \quad \forall f \in L^{2}\left(\mathbb{R}^{2}\right) .
$$

Copyright 2016 by the Tusi Mathematical Research Group.

Received Sep. 21, 2015; Accepted Dec. 15, 2015.

2010 Mathematics Subject Classification. Primary 46N99; Secondary 47N99, 46E99, 42C40.

Keywords. Fourier transform, Hilbert space, expansive integral matrix, normalized tight frame wavelet. 


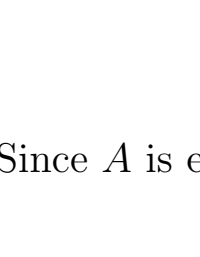

Banach J. Math. Anal. 10 (2016), no. 3, 638-670

http://dx.doi.org/10.1215/17358787-3639712

ISSN: $1735-8787$ (electronic)

http://projecteuclid.org/bjma

\title{
EQUATIONS FOR FRAME WAVELETS IN $L^{2}\left(\mathbb{R}^{2}\right)$
}

\author{
XINGDE DAI \\ To Professor Zurui Guo \\ Communicated by D. Han
}

\begin{abstract}
A finite solution to a system of equations will generate a single function normalized tight frame wavelet (Parseval's frame wavelet) with compact support associated with a $2 \times 2$ expansive integral matrix whose determinant is either 2 or -2 in $L^{2}\left(\mathbb{R}^{2}\right)$.
\end{abstract}

\section{IntRoduction}

In this article, $\mathbb{R}^{2}$ will be the 2-dimensional Euclidean space, and $\mathbb{C}^{2}$ will be the 2-dimensional complex Euclidean space. We will use notation $\vec{t}, \vec{s}, \vec{r}, \vec{\xi}, \vec{\eta}$ for vectors in $\mathbb{R}^{2}$ or $\mathbb{C}^{2}$. We will use the notation $\vec{t}_{1} \circ \vec{t}_{2}$ for the standard inner product of two vectors $\vec{t}_{1}$ and $\vec{t}_{2}$. For a vector $\vec{\xi}=\left(\begin{array}{c}\xi_{1} \\ \xi_{2}\end{array}\right)$ in $\mathbb{C}^{2}$, its real part is $\mathfrak{R e}(\vec{\xi}) \equiv\left(\begin{array}{c}\mathfrak{R e}\left(\xi_{1}\right) \\ \mathfrak{R}\left(\xi_{2}\right)\end{array}\right)$, and its imaginary part is $\mathfrak{I m}(\vec{\xi}) \equiv\left(\begin{array}{c}\mathfrak{I m}\left(\xi_{1}\right) \\ \mathfrak{I m}\left(\xi_{2}\right)\end{array}\right)$. The measure $\mu$ will be the Lebesgue measure on $\mathbb{R}^{2}$, and $L^{2}\left(\mathbb{R}^{2}\right)$ will be the Hilbert space of all square integrable functions on $\mathbb{R}^{2}$. A (countable) set of elements $\left\{\psi_{i}: i \in \Lambda\right\}$ in $L^{2}\left(\mathbb{R}^{2}\right)$ is called a normalized tight frame of $L^{2}\left(\mathbb{R}^{2}\right)$ if

$$
\sum_{i \in \Lambda}\left|\left\langle f, \psi_{i}\right\rangle\right|^{2}=\|f\|^{2}, \quad \forall f \in L^{2}\left(\mathbb{R}^{2}\right) .
$$

It is well known in the literature (see [4]) that the equation (1.1) is equivalent to

$$
f=\sum_{i \in \Lambda}\left\langle f, \psi_{i}\right\rangle \psi_{i}, \quad \forall f \in L^{2}\left(\mathbb{R}^{2}\right) .
$$

Copyright 2016 by the Tusi Mathematical Research Group.

Received Sep. 21, 2015; Accepted Dec. 15, 2015.

2010 Mathematics Subject Classification. Primary 46N99; Secondary 47N99, 46E99, 42C40.

Keywords. Fourier transform, Hilbert space, expansive integral matrix, normalized tight frame wavelet. 


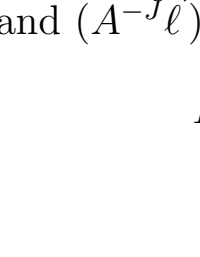

Banach J. Math. Anal. 10 (2016), no. 3, 638-670

http://dx.doi.org/10.1215/17358787-3639712

ISSN: $1735-8787$ (electronic)

http://projecteuclid.org/bjma

\title{
EQUATIONS FOR FRAME WAVELETS IN $L^{2}\left(\mathbb{R}^{2}\right)$
}

\author{
XINGDE DAI \\ To Professor Zurui Guo \\ Communicated by D. Han
}

\begin{abstract}
A finite solution to a system of equations will generate a single function normalized tight frame wavelet (Parseval's frame wavelet) with compact support associated with a $2 \times 2$ expansive integral matrix whose determinant is either 2 or -2 in $L^{2}\left(\mathbb{R}^{2}\right)$.
\end{abstract}

\section{IntRoduction}

In this article, $\mathbb{R}^{2}$ will be the 2-dimensional Euclidean space, and $\mathbb{C}^{2}$ will be the 2-dimensional complex Euclidean space. We will use notation $\vec{t}, \vec{s}, \vec{r}, \vec{\xi}, \vec{\eta}$ for vectors in $\mathbb{R}^{2}$ or $\mathbb{C}^{2}$. We will use the notation $\vec{t}_{1} \circ \vec{t}_{2}$ for the standard inner product of two vectors $\vec{t}_{1}$ and $\vec{t}_{2}$. For a vector $\vec{\xi}=\left(\begin{array}{c}\xi_{1} \\ \xi_{2}\end{array}\right)$ in $\mathbb{C}^{2}$, its real part is $\mathfrak{R e}(\vec{\xi}) \equiv\left(\begin{array}{c}\mathfrak{R e}\left(\xi_{1}\right) \\ \mathfrak{R}\left(\xi_{2}\right)\end{array}\right)$, and its imaginary part is $\mathfrak{I m}(\vec{\xi}) \equiv\left(\begin{array}{c}\mathfrak{I m}\left(\xi_{1}\right) \\ \mathfrak{I m}\left(\xi_{2}\right)\end{array}\right)$. The measure $\mu$ will be the Lebesgue measure on $\mathbb{R}^{2}$, and $L^{2}\left(\mathbb{R}^{2}\right)$ will be the Hilbert space of all square integrable functions on $\mathbb{R}^{2}$. A (countable) set of elements $\left\{\psi_{i}: i \in \Lambda\right\}$ in $L^{2}\left(\mathbb{R}^{2}\right)$ is called a normalized tight frame of $L^{2}\left(\mathbb{R}^{2}\right)$ if

$$
\sum_{i \in \Lambda}\left|\left\langle f, \psi_{i}\right\rangle\right|^{2}=\|f\|^{2}, \quad \forall f \in L^{2}\left(\mathbb{R}^{2}\right) .
$$

It is well known in the literature (see [4]) that the equation (1.1) is equivalent to

$$
f=\sum_{i \in \Lambda}\left\langle f, \psi_{i}\right\rangle \psi_{i}, \quad \forall f \in L^{2}\left(\mathbb{R}^{2}\right) .
$$

Copyright 2016 by the Tusi Mathematical Research Group.

Received Sep. 21, 2015; Accepted Dec. 15, 2015.

2010 Mathematics Subject Classification. Primary 46N99; Secondary 47N99, 46E99, 42C40.

Keywords. Fourier transform, Hilbert space, expansive integral matrix, normalized tight frame wavelet. 


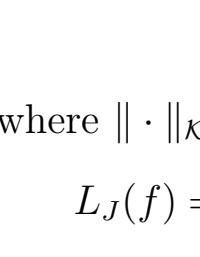

Banach J. Math. Anal. 10 (2016), no. 3, 638-670

http://dx.doi.org/10.1215/17358787-3639712

ISSN: $1735-8787$ (electronic)

http://projecteuclid.org/bjma

\title{
EQUATIONS FOR FRAME WAVELETS IN $L^{2}\left(\mathbb{R}^{2}\right)$
}

\author{
XINGDE DAI \\ To Professor Zurui Guo \\ Communicated by D. Han
}

\begin{abstract}
A finite solution to a system of equations will generate a single function normalized tight frame wavelet (Parseval's frame wavelet) with compact support associated with a $2 \times 2$ expansive integral matrix whose determinant is either 2 or -2 in $L^{2}\left(\mathbb{R}^{2}\right)$.
\end{abstract}

\section{IntRoduction}

In this article, $\mathbb{R}^{2}$ will be the 2-dimensional Euclidean space, and $\mathbb{C}^{2}$ will be the 2-dimensional complex Euclidean space. We will use notation $\vec{t}, \vec{s}, \vec{r}, \vec{\xi}, \vec{\eta}$ for vectors in $\mathbb{R}^{2}$ or $\mathbb{C}^{2}$. We will use the notation $\vec{t}_{1} \circ \vec{t}_{2}$ for the standard inner product of two vectors $\vec{t}_{1}$ and $\vec{t}_{2}$. For a vector $\vec{\xi}=\left(\begin{array}{c}\xi_{1} \\ \xi_{2}\end{array}\right)$ in $\mathbb{C}^{2}$, its real part is $\mathfrak{R e}(\vec{\xi}) \equiv\left(\begin{array}{c}\mathfrak{R e}\left(\xi_{1}\right) \\ \mathfrak{R}\left(\xi_{2}\right)\end{array}\right)$, and its imaginary part is $\mathfrak{I m}(\vec{\xi}) \equiv\left(\begin{array}{c}\mathfrak{I m}\left(\xi_{1}\right) \\ \mathfrak{I m}\left(\xi_{2}\right)\end{array}\right)$. The measure $\mu$ will be the Lebesgue measure on $\mathbb{R}^{2}$, and $L^{2}\left(\mathbb{R}^{2}\right)$ will be the Hilbert space of all square integrable functions on $\mathbb{R}^{2}$. A (countable) set of elements $\left\{\psi_{i}: i \in \Lambda\right\}$ in $L^{2}\left(\mathbb{R}^{2}\right)$ is called a normalized tight frame of $L^{2}\left(\mathbb{R}^{2}\right)$ if

$$
\sum_{i \in \Lambda}\left|\left\langle f, \psi_{i}\right\rangle\right|^{2}=\|f\|^{2}, \quad \forall f \in L^{2}\left(\mathbb{R}^{2}\right) .
$$

It is well known in the literature (see [4]) that the equation (1.1) is equivalent to

$$
f=\sum_{i \in \Lambda}\left\langle f, \psi_{i}\right\rangle \psi_{i}, \quad \forall f \in L^{2}\left(\mathbb{R}^{2}\right) .
$$

Copyright 2016 by the Tusi Mathematical Research Group.

Received Sep. 21, 2015; Accepted Dec. 15, 2015.

2010 Mathematics Subject Classification. Primary 46N99; Secondary 47N99, 46E99, 42C40.

Keywords. Fourier transform, Hilbert space, expansive integral matrix, normalized tight frame wavelet. 


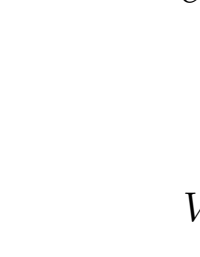

Banach J. Math. Anal. 10 (2016), no. 3, 638-670

http://dx.doi.org/10.1215/17358787-3639712

ISSN: $1735-8787$ (electronic)

http://projecteuclid.org/bjma

\title{
EQUATIONS FOR FRAME WAVELETS IN $L^{2}\left(\mathbb{R}^{2}\right)$
}

\author{
XINGDE DAI \\ To Professor Zurui Guo \\ Communicated by D. Han
}

\begin{abstract}
A finite solution to a system of equations will generate a single function normalized tight frame wavelet (Parseval's frame wavelet) with compact support associated with a $2 \times 2$ expansive integral matrix whose determinant is either 2 or -2 in $L^{2}\left(\mathbb{R}^{2}\right)$.
\end{abstract}

\section{IntRoduction}

In this article, $\mathbb{R}^{2}$ will be the 2-dimensional Euclidean space, and $\mathbb{C}^{2}$ will be the 2-dimensional complex Euclidean space. We will use notation $\vec{t}, \vec{s}, \vec{r}, \vec{\xi}, \vec{\eta}$ for vectors in $\mathbb{R}^{2}$ or $\mathbb{C}^{2}$. We will use the notation $\vec{t}_{1} \circ \vec{t}_{2}$ for the standard inner product of two vectors $\vec{t}_{1}$ and $\vec{t}_{2}$. For a vector $\vec{\xi}=\left(\begin{array}{c}\xi_{1} \\ \xi_{2}\end{array}\right)$ in $\mathbb{C}^{2}$, its real part is $\mathfrak{R e}(\vec{\xi}) \equiv\left(\begin{array}{c}\mathfrak{R e}\left(\xi_{1}\right) \\ \mathfrak{R}\left(\xi_{2}\right)\end{array}\right)$, and its imaginary part is $\mathfrak{I m}(\vec{\xi}) \equiv\left(\begin{array}{c}\mathfrak{I m}\left(\xi_{1}\right) \\ \mathfrak{I m}\left(\xi_{2}\right)\end{array}\right)$. The measure $\mu$ will be the Lebesgue measure on $\mathbb{R}^{2}$, and $L^{2}\left(\mathbb{R}^{2}\right)$ will be the Hilbert space of all square integrable functions on $\mathbb{R}^{2}$. A (countable) set of elements $\left\{\psi_{i}: i \in \Lambda\right\}$ in $L^{2}\left(\mathbb{R}^{2}\right)$ is called a normalized tight frame of $L^{2}\left(\mathbb{R}^{2}\right)$ if

$$
\sum_{i \in \Lambda}\left|\left\langle f, \psi_{i}\right\rangle\right|^{2}=\|f\|^{2}, \quad \forall f \in L^{2}\left(\mathbb{R}^{2}\right) .
$$

It is well known in the literature (see [4]) that the equation (1.1) is equivalent to

$$
f=\sum_{i \in \Lambda}\left\langle f, \psi_{i}\right\rangle \psi_{i}, \quad \forall f \in L^{2}\left(\mathbb{R}^{2}\right) .
$$

Copyright 2016 by the Tusi Mathematical Research Group.

Received Sep. 21, 2015; Accepted Dec. 15, 2015.

2010 Mathematics Subject Classification. Primary 46N99; Secondary 47N99, 46E99, 42C40.

Keywords. Fourier transform, Hilbert space, expansive integral matrix, normalized tight frame wavelet. 


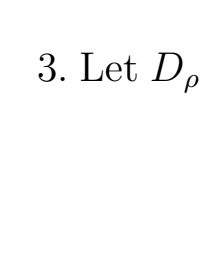

Banach J. Math. Anal. 10 (2016), no. 3, 638-670

http://dx.doi.org/10.1215/17358787-3639712

ISSN: $1735-8787$ (electronic)

http://projecteuclid.org/bjma

\title{
EQUATIONS FOR FRAME WAVELETS IN $L^{2}\left(\mathbb{R}^{2}\right)$
}

\author{
XINGDE DAI \\ To Professor Zurui Guo \\ Communicated by D. Han
}

\begin{abstract}
A finite solution to a system of equations will generate a single function normalized tight frame wavelet (Parseval's frame wavelet) with compact support associated with a $2 \times 2$ expansive integral matrix whose determinant is either 2 or -2 in $L^{2}\left(\mathbb{R}^{2}\right)$.
\end{abstract}

\section{IntRoduction}

In this article, $\mathbb{R}^{2}$ will be the 2-dimensional Euclidean space, and $\mathbb{C}^{2}$ will be the 2-dimensional complex Euclidean space. We will use notation $\vec{t}, \vec{s}, \vec{r}, \vec{\xi}, \vec{\eta}$ for vectors in $\mathbb{R}^{2}$ or $\mathbb{C}^{2}$. We will use the notation $\vec{t}_{1} \circ \vec{t}_{2}$ for the standard inner product of two vectors $\vec{t}_{1}$ and $\vec{t}_{2}$. For a vector $\vec{\xi}=\left(\begin{array}{c}\xi_{1} \\ \xi_{2}\end{array}\right)$ in $\mathbb{C}^{2}$, its real part is $\mathfrak{R e}(\vec{\xi}) \equiv\left(\begin{array}{c}\mathfrak{R e}\left(\xi_{1}\right) \\ \mathfrak{R}\left(\xi_{2}\right)\end{array}\right)$, and its imaginary part is $\mathfrak{I m}(\vec{\xi}) \equiv\left(\begin{array}{c}\mathfrak{I m}\left(\xi_{1}\right) \\ \mathfrak{I m}\left(\xi_{2}\right)\end{array}\right)$. The measure $\mu$ will be the Lebesgue measure on $\mathbb{R}^{2}$, and $L^{2}\left(\mathbb{R}^{2}\right)$ will be the Hilbert space of all square integrable functions on $\mathbb{R}^{2}$. A (countable) set of elements $\left\{\psi_{i}: i \in \Lambda\right\}$ in $L^{2}\left(\mathbb{R}^{2}\right)$ is called a normalized tight frame of $L^{2}\left(\mathbb{R}^{2}\right)$ if

$$
\sum_{i \in \Lambda}\left|\left\langle f, \psi_{i}\right\rangle\right|^{2}=\|f\|^{2}, \quad \forall f \in L^{2}\left(\mathbb{R}^{2}\right) .
$$

It is well known in the literature (see [4]) that the equation (1.1) is equivalent to

$$
f=\sum_{i \in \Lambda}\left\langle f, \psi_{i}\right\rangle \psi_{i}, \quad \forall f \in L^{2}\left(\mathbb{R}^{2}\right) .
$$

Copyright 2016 by the Tusi Mathematical Research Group.

Received Sep. 21, 2015; Accepted Dec. 15, 2015.

2010 Mathematics Subject Classification. Primary 46N99; Secondary 47N99, 46E99, 42C40.

Keywords. Fourier transform, Hilbert space, expansive integral matrix, normalized tight frame wavelet. 


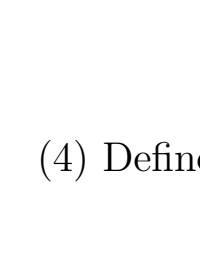

Banach J. Math. Anal. 10 (2016), no. 3, 638-670

http://dx.doi.org/10.1215/17358787-3639712

ISSN: $1735-8787$ (electronic)

http://projecteuclid.org/bjma

\title{
EQUATIONS FOR FRAME WAVELETS IN $L^{2}\left(\mathbb{R}^{2}\right)$
}

\author{
XINGDE DAI \\ To Professor Zurui Guo \\ Communicated by D. Han
}

\begin{abstract}
A finite solution to a system of equations will generate a single function normalized tight frame wavelet (Parseval's frame wavelet) with compact support associated with a $2 \times 2$ expansive integral matrix whose determinant is either 2 or -2 in $L^{2}\left(\mathbb{R}^{2}\right)$.
\end{abstract}

\section{IntRoduction}

In this article, $\mathbb{R}^{2}$ will be the 2-dimensional Euclidean space, and $\mathbb{C}^{2}$ will be the 2-dimensional complex Euclidean space. We will use notation $\vec{t}, \vec{s}, \vec{r}, \vec{\xi}, \vec{\eta}$ for vectors in $\mathbb{R}^{2}$ or $\mathbb{C}^{2}$. We will use the notation $\vec{t}_{1} \circ \vec{t}_{2}$ for the standard inner product of two vectors $\vec{t}_{1}$ and $\vec{t}_{2}$. For a vector $\vec{\xi}=\left(\begin{array}{c}\xi_{1} \\ \xi_{2}\end{array}\right)$ in $\mathbb{C}^{2}$, its real part is $\mathfrak{R e}(\vec{\xi}) \equiv\left(\begin{array}{c}\mathfrak{R e}\left(\xi_{1}\right) \\ \mathfrak{R}\left(\xi_{2}\right)\end{array}\right)$, and its imaginary part is $\mathfrak{I m}(\vec{\xi}) \equiv\left(\begin{array}{c}\mathfrak{I m}\left(\xi_{1}\right) \\ \mathfrak{I m}\left(\xi_{2}\right)\end{array}\right)$. The measure $\mu$ will be the Lebesgue measure on $\mathbb{R}^{2}$, and $L^{2}\left(\mathbb{R}^{2}\right)$ will be the Hilbert space of all square integrable functions on $\mathbb{R}^{2}$. A (countable) set of elements $\left\{\psi_{i}: i \in \Lambda\right\}$ in $L^{2}\left(\mathbb{R}^{2}\right)$ is called a normalized tight frame of $L^{2}\left(\mathbb{R}^{2}\right)$ if

$$
\sum_{i \in \Lambda}\left|\left\langle f, \psi_{i}\right\rangle\right|^{2}=\|f\|^{2}, \quad \forall f \in L^{2}\left(\mathbb{R}^{2}\right) .
$$

It is well known in the literature (see [4]) that the equation (1.1) is equivalent to

$$
f=\sum_{i \in \Lambda}\left\langle f, \psi_{i}\right\rangle \psi_{i}, \quad \forall f \in L^{2}\left(\mathbb{R}^{2}\right) .
$$

Copyright 2016 by the Tusi Mathematical Research Group.

Received Sep. 21, 2015; Accepted Dec. 15, 2015.

2010 Mathematics Subject Classification. Primary 46N99; Secondary 47N99, 46E99, 42C40.

Keywords. Fourier transform, Hilbert space, expansive integral matrix, normalized tight frame wavelet. 


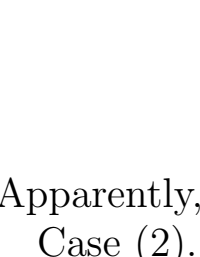

Banach J. Math. Anal. 10 (2016), no. 3, 638-670

http://dx.doi.org/10.1215/17358787-3639712

ISSN: $1735-8787$ (electronic)

http://projecteuclid.org/bjma

\title{
EQUATIONS FOR FRAME WAVELETS IN $L^{2}\left(\mathbb{R}^{2}\right)$
}

\author{
XINGDE DAI \\ To Professor Zurui Guo \\ Communicated by D. Han
}

\begin{abstract}
A finite solution to a system of equations will generate a single function normalized tight frame wavelet (Parseval's frame wavelet) with compact support associated with a $2 \times 2$ expansive integral matrix whose determinant is either 2 or -2 in $L^{2}\left(\mathbb{R}^{2}\right)$.
\end{abstract}

\section{IntRoduction}

In this article, $\mathbb{R}^{2}$ will be the 2-dimensional Euclidean space, and $\mathbb{C}^{2}$ will be the 2-dimensional complex Euclidean space. We will use notation $\vec{t}, \vec{s}, \vec{r}, \vec{\xi}, \vec{\eta}$ for vectors in $\mathbb{R}^{2}$ or $\mathbb{C}^{2}$. We will use the notation $\vec{t}_{1} \circ \vec{t}_{2}$ for the standard inner product of two vectors $\vec{t}_{1}$ and $\vec{t}_{2}$. For a vector $\vec{\xi}=\left(\begin{array}{c}\xi_{1} \\ \xi_{2}\end{array}\right)$ in $\mathbb{C}^{2}$, its real part is $\mathfrak{R e}(\vec{\xi}) \equiv\left(\begin{array}{c}\mathfrak{R e}\left(\xi_{1}\right) \\ \mathfrak{R}\left(\xi_{2}\right)\end{array}\right)$, and its imaginary part is $\mathfrak{I m}(\vec{\xi}) \equiv\left(\begin{array}{c}\mathfrak{I m}\left(\xi_{1}\right) \\ \mathfrak{I m}\left(\xi_{2}\right)\end{array}\right)$. The measure $\mu$ will be the Lebesgue measure on $\mathbb{R}^{2}$, and $L^{2}\left(\mathbb{R}^{2}\right)$ will be the Hilbert space of all square integrable functions on $\mathbb{R}^{2}$. A (countable) set of elements $\left\{\psi_{i}: i \in \Lambda\right\}$ in $L^{2}\left(\mathbb{R}^{2}\right)$ is called a normalized tight frame of $L^{2}\left(\mathbb{R}^{2}\right)$ if

$$
\sum_{i \in \Lambda}\left|\left\langle f, \psi_{i}\right\rangle\right|^{2}=\|f\|^{2}, \quad \forall f \in L^{2}\left(\mathbb{R}^{2}\right) .
$$

It is well known in the literature (see [4]) that the equation (1.1) is equivalent to

$$
f=\sum_{i \in \Lambda}\left\langle f, \psi_{i}\right\rangle \psi_{i}, \quad \forall f \in L^{2}\left(\mathbb{R}^{2}\right) .
$$

Copyright 2016 by the Tusi Mathematical Research Group.

Received Sep. 21, 2015; Accepted Dec. 15, 2015.

2010 Mathematics Subject Classification. Primary 46N99; Secondary 47N99, 46E99, 42C40.

Keywords. Fourier transform, Hilbert space, expansive integral matrix, normalized tight frame wavelet. 


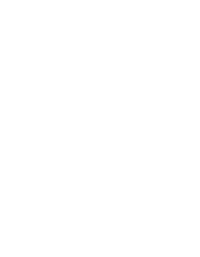

Banach J. Math. Anal. 10 (2016), no. 3, 638-670

http://dx.doi.org/10.1215/17358787-3639712

ISSN: $1735-8787$ (electronic)

http://projecteuclid.org/bjma

\title{
EQUATIONS FOR FRAME WAVELETS IN $L^{2}\left(\mathbb{R}^{2}\right)$
}

\author{
XINGDE DAI \\ To Professor Zurui Guo \\ Communicated by D. Han
}

\begin{abstract}
A finite solution to a system of equations will generate a single function normalized tight frame wavelet (Parseval's frame wavelet) with compact support associated with a $2 \times 2$ expansive integral matrix whose determinant is either 2 or -2 in $L^{2}\left(\mathbb{R}^{2}\right)$.
\end{abstract}

\section{IntRoduction}

In this article, $\mathbb{R}^{2}$ will be the 2-dimensional Euclidean space, and $\mathbb{C}^{2}$ will be the 2-dimensional complex Euclidean space. We will use notation $\vec{t}, \vec{s}, \vec{r}, \vec{\xi}, \vec{\eta}$ for vectors in $\mathbb{R}^{2}$ or $\mathbb{C}^{2}$. We will use the notation $\vec{t}_{1} \circ \vec{t}_{2}$ for the standard inner product of two vectors $\vec{t}_{1}$ and $\vec{t}_{2}$. For a vector $\vec{\xi}=\left(\begin{array}{c}\xi_{1} \\ \xi_{2}\end{array}\right)$ in $\mathbb{C}^{2}$, its real part is $\mathfrak{R e}(\vec{\xi}) \equiv\left(\begin{array}{c}\mathfrak{R e}\left(\xi_{1}\right) \\ \mathfrak{R}\left(\xi_{2}\right)\end{array}\right)$, and its imaginary part is $\mathfrak{I m}(\vec{\xi}) \equiv\left(\begin{array}{c}\mathfrak{I m}\left(\xi_{1}\right) \\ \mathfrak{I m}\left(\xi_{2}\right)\end{array}\right)$. The measure $\mu$ will be the Lebesgue measure on $\mathbb{R}^{2}$, and $L^{2}\left(\mathbb{R}^{2}\right)$ will be the Hilbert space of all square integrable functions on $\mathbb{R}^{2}$. A (countable) set of elements $\left\{\psi_{i}: i \in \Lambda\right\}$ in $L^{2}\left(\mathbb{R}^{2}\right)$ is called a normalized tight frame of $L^{2}\left(\mathbb{R}^{2}\right)$ if

$$
\sum_{i \in \Lambda}\left|\left\langle f, \psi_{i}\right\rangle\right|^{2}=\|f\|^{2}, \quad \forall f \in L^{2}\left(\mathbb{R}^{2}\right) .
$$

It is well known in the literature (see [4]) that the equation (1.1) is equivalent to

$$
f=\sum_{i \in \Lambda}\left\langle f, \psi_{i}\right\rangle \psi_{i}, \quad \forall f \in L^{2}\left(\mathbb{R}^{2}\right) .
$$

Copyright 2016 by the Tusi Mathematical Research Group.

Received Sep. 21, 2015; Accepted Dec. 15, 2015.

2010 Mathematics Subject Classification. Primary 46N99; Secondary 47N99, 46E99, 42C40.

Keywords. Fourier transform, Hilbert space, expansive integral matrix, normalized tight frame wavelet. 


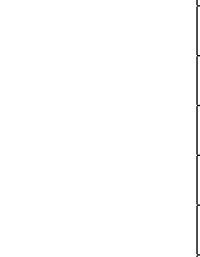

Banach J. Math. Anal. 10 (2016), no. 3, 638-670

http://dx.doi.org/10.1215/17358787-3639712

ISSN: $1735-8787$ (electronic)

http://projecteuclid.org/bjma

\title{
EQUATIONS FOR FRAME WAVELETS IN $L^{2}\left(\mathbb{R}^{2}\right)$
}

\author{
XINGDE DAI \\ To Professor Zurui Guo \\ Communicated by D. Han
}

\begin{abstract}
A finite solution to a system of equations will generate a single function normalized tight frame wavelet (Parseval's frame wavelet) with compact support associated with a $2 \times 2$ expansive integral matrix whose determinant is either 2 or -2 in $L^{2}\left(\mathbb{R}^{2}\right)$.
\end{abstract}

\section{IntRoduction}

In this article, $\mathbb{R}^{2}$ will be the 2-dimensional Euclidean space, and $\mathbb{C}^{2}$ will be the 2-dimensional complex Euclidean space. We will use notation $\vec{t}, \vec{s}, \vec{r}, \vec{\xi}, \vec{\eta}$ for vectors in $\mathbb{R}^{2}$ or $\mathbb{C}^{2}$. We will use the notation $\vec{t}_{1} \circ \vec{t}_{2}$ for the standard inner product of two vectors $\vec{t}_{1}$ and $\vec{t}_{2}$. For a vector $\vec{\xi}=\left(\begin{array}{c}\xi_{1} \\ \xi_{2}\end{array}\right)$ in $\mathbb{C}^{2}$, its real part is $\mathfrak{R e}(\vec{\xi}) \equiv\left(\begin{array}{c}\mathfrak{R e}\left(\xi_{1}\right) \\ \mathfrak{R}\left(\xi_{2}\right)\end{array}\right)$, and its imaginary part is $\mathfrak{I m}(\vec{\xi}) \equiv\left(\begin{array}{c}\mathfrak{I m}\left(\xi_{1}\right) \\ \mathfrak{I m}\left(\xi_{2}\right)\end{array}\right)$. The measure $\mu$ will be the Lebesgue measure on $\mathbb{R}^{2}$, and $L^{2}\left(\mathbb{R}^{2}\right)$ will be the Hilbert space of all square integrable functions on $\mathbb{R}^{2}$. A (countable) set of elements $\left\{\psi_{i}: i \in \Lambda\right\}$ in $L^{2}\left(\mathbb{R}^{2}\right)$ is called a normalized tight frame of $L^{2}\left(\mathbb{R}^{2}\right)$ if

$$
\sum_{i \in \Lambda}\left|\left\langle f, \psi_{i}\right\rangle\right|^{2}=\|f\|^{2}, \quad \forall f \in L^{2}\left(\mathbb{R}^{2}\right) .
$$

It is well known in the literature (see [4]) that the equation (1.1) is equivalent to

$$
f=\sum_{i \in \Lambda}\left\langle f, \psi_{i}\right\rangle \psi_{i}, \quad \forall f \in L^{2}\left(\mathbb{R}^{2}\right) .
$$

Copyright 2016 by the Tusi Mathematical Research Group.

Received Sep. 21, 2015; Accepted Dec. 15, 2015.

2010 Mathematics Subject Classification. Primary 46N99; Secondary 47N99, 46E99, 42C40.

Keywords. Fourier transform, Hilbert space, expansive integral matrix, normalized tight frame wavelet. 


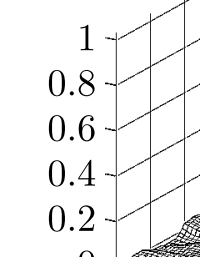

Banach J. Math. Anal. 10 (2016), no. 3, 638-670

http://dx.doi.org/10.1215/17358787-3639712

ISSN: $1735-8787$ (electronic)

http://projecteuclid.org/bjma

\title{
EQUATIONS FOR FRAME WAVELETS IN $L^{2}\left(\mathbb{R}^{2}\right)$
}

\author{
XINGDE DAI \\ To Professor Zurui Guo \\ Communicated by D. Han
}

\begin{abstract}
A finite solution to a system of equations will generate a single function normalized tight frame wavelet (Parseval's frame wavelet) with compact support associated with a $2 \times 2$ expansive integral matrix whose determinant is either 2 or -2 in $L^{2}\left(\mathbb{R}^{2}\right)$.
\end{abstract}

\section{IntRoduction}

In this article, $\mathbb{R}^{2}$ will be the 2-dimensional Euclidean space, and $\mathbb{C}^{2}$ will be the 2-dimensional complex Euclidean space. We will use notation $\vec{t}, \vec{s}, \vec{r}, \vec{\xi}, \vec{\eta}$ for vectors in $\mathbb{R}^{2}$ or $\mathbb{C}^{2}$. We will use the notation $\vec{t}_{1} \circ \vec{t}_{2}$ for the standard inner product of two vectors $\vec{t}_{1}$ and $\vec{t}_{2}$. For a vector $\vec{\xi}=\left(\begin{array}{c}\xi_{1} \\ \xi_{2}\end{array}\right)$ in $\mathbb{C}^{2}$, its real part is $\mathfrak{R e}(\vec{\xi}) \equiv\left(\begin{array}{c}\mathfrak{R e}\left(\xi_{1}\right) \\ \mathfrak{R}\left(\xi_{2}\right)\end{array}\right)$, and its imaginary part is $\mathfrak{I m}(\vec{\xi}) \equiv\left(\begin{array}{c}\mathfrak{I m}\left(\xi_{1}\right) \\ \mathfrak{I m}\left(\xi_{2}\right)\end{array}\right)$. The measure $\mu$ will be the Lebesgue measure on $\mathbb{R}^{2}$, and $L^{2}\left(\mathbb{R}^{2}\right)$ will be the Hilbert space of all square integrable functions on $\mathbb{R}^{2}$. A (countable) set of elements $\left\{\psi_{i}: i \in \Lambda\right\}$ in $L^{2}\left(\mathbb{R}^{2}\right)$ is called a normalized tight frame of $L^{2}\left(\mathbb{R}^{2}\right)$ if

$$
\sum_{i \in \Lambda}\left|\left\langle f, \psi_{i}\right\rangle\right|^{2}=\|f\|^{2}, \quad \forall f \in L^{2}\left(\mathbb{R}^{2}\right) .
$$

It is well known in the literature (see [4]) that the equation (1.1) is equivalent to

$$
f=\sum_{i \in \Lambda}\left\langle f, \psi_{i}\right\rangle \psi_{i}, \quad \forall f \in L^{2}\left(\mathbb{R}^{2}\right) .
$$

Copyright 2016 by the Tusi Mathematical Research Group.

Received Sep. 21, 2015; Accepted Dec. 15, 2015.

2010 Mathematics Subject Classification. Primary 46N99; Secondary 47N99, 46E99, 42C40.

Keywords. Fourier transform, Hilbert space, expansive integral matrix, normalized tight frame wavelet. 


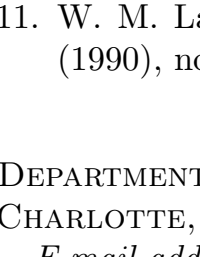

Banach J. Math. Anal. 10 (2016), no. 3, 638-670

http://dx.doi.org/10.1215/17358787-3639712

ISSN: $1735-8787$ (electronic)

http://projecteuclid.org/bjma

\title{
EQUATIONS FOR FRAME WAVELETS IN $L^{2}\left(\mathbb{R}^{2}\right)$
}

\author{
XINGDE DAI \\ To Professor Zurui Guo \\ Communicated by D. Han
}

\begin{abstract}
A finite solution to a system of equations will generate a single function normalized tight frame wavelet (Parseval's frame wavelet) with compact support associated with a $2 \times 2$ expansive integral matrix whose determinant is either 2 or -2 in $L^{2}\left(\mathbb{R}^{2}\right)$.
\end{abstract}

\section{IntRoduction}

In this article, $\mathbb{R}^{2}$ will be the 2-dimensional Euclidean space, and $\mathbb{C}^{2}$ will be the 2-dimensional complex Euclidean space. We will use notation $\vec{t}, \vec{s}, \vec{r}, \vec{\xi}, \vec{\eta}$ for vectors in $\mathbb{R}^{2}$ or $\mathbb{C}^{2}$. We will use the notation $\vec{t}_{1} \circ \vec{t}_{2}$ for the standard inner product of two vectors $\vec{t}_{1}$ and $\vec{t}_{2}$. For a vector $\vec{\xi}=\left(\begin{array}{c}\xi_{1} \\ \xi_{2}\end{array}\right)$ in $\mathbb{C}^{2}$, its real part is $\mathfrak{R e}(\vec{\xi}) \equiv\left(\begin{array}{c}\mathfrak{R e}\left(\xi_{1}\right) \\ \mathfrak{R}\left(\xi_{2}\right)\end{array}\right)$, and its imaginary part is $\mathfrak{I m}(\vec{\xi}) \equiv\left(\begin{array}{c}\mathfrak{I m}\left(\xi_{1}\right) \\ \mathfrak{I m}\left(\xi_{2}\right)\end{array}\right)$. The measure $\mu$ will be the Lebesgue measure on $\mathbb{R}^{2}$, and $L^{2}\left(\mathbb{R}^{2}\right)$ will be the Hilbert space of all square integrable functions on $\mathbb{R}^{2}$. A (countable) set of elements $\left\{\psi_{i}: i \in \Lambda\right\}$ in $L^{2}\left(\mathbb{R}^{2}\right)$ is called a normalized tight frame of $L^{2}\left(\mathbb{R}^{2}\right)$ if

$$
\sum_{i \in \Lambda}\left|\left\langle f, \psi_{i}\right\rangle\right|^{2}=\|f\|^{2}, \quad \forall f \in L^{2}\left(\mathbb{R}^{2}\right) .
$$

It is well known in the literature (see [4]) that the equation (1.1) is equivalent to

$$
f=\sum_{i \in \Lambda}\left\langle f, \psi_{i}\right\rangle \psi_{i}, \quad \forall f \in L^{2}\left(\mathbb{R}^{2}\right) .
$$

Copyright 2016 by the Tusi Mathematical Research Group.

Received Sep. 21, 2015; Accepted Dec. 15, 2015.

2010 Mathematics Subject Classification. Primary 46N99; Secondary 47N99, 46E99, 42C40.

Keywords. Fourier transform, Hilbert space, expansive integral matrix, normalized tight frame wavelet. 